\title{
Consensus Control of Linear Multi-agent Systems under Actuator Imperfection: When Saturation Meets Fault
}

\author{
Chen Gao, Zidong Wang, Fellow, IEEE, Xiao He, Senior Member, IEEE, and Qing-Long Han, Fellow, IEEE
}

\begin{abstract}
This paper is concerned with the consensus control problem for a general class of linear multi-agent systems (MASs) subject to actuator imperfection consisting of both actuator saturations and actuator faults. A novel two-step saturation-resistant approach is proposed to attenuate the side effects resulting from the actuator imperfection. In the first step of controller design, the state information received from the neighboring agents is used to design a consensus controller capable of tolerating the actuator fault. Then, in the second step of controller optimization, the domain of attraction (DOA) is introduced for MASs to evaluate the performance of the controller and, subsequently, optimize the controller parameters to enlarge the DOA in terms of solutions to a certain set of matrix inequalities. Finally, simulation examples are provided to demonstrate the effectiveness of the developed saturation-resistant approach.
\end{abstract}

Index Terms-Fault-tolerant control, adaptive control, multiagent system, actuator saturation, leaderless consensus, leaderfollower consensus.

\section{INTRODUCTION}

Multi-agent systems (MASs), which include unmanned aerial vehicle (UAV) systems [38] and flocking systems [17] as typical examples, consist of multiple interacting agents capable of accomplishing complicated missions that are difficult (if not impossible) for a single agent to achieve. As one of the main-stream research topics with MASs, the consensus control problem has received an ever-increasing research interest and the relevant literature can be dated back to [10], [30], [31], where the connection among agents has been modeled as a topology graph and a series of basic consensus controllers have been designed. In the past few years, the consensus control problem has continued to attract a great deal of research attention with a series of excellent results reported [9], [12], [21], [23], [43], and the main research focuses have been

This work was supported in part by the National Natural Science Foundation of China under Grants 61873148 and 61733009, the National Key Research and Development Program of China under Grant 2017YFA0700300, the Natural Science Foundation of Guangdong Province of China under Grant 2018B030311054, the Beijing National Research Center for Information Science and Technology (BNRist) Program of China under Grant BNR2019TD01009, the Royal Society of the UK, and the Alexander von Humboldt Foundation of Germany. (Corresponding author: Xiao He.)

$\mathrm{X}$. He and C. Gao are with the Department of Automation, BNRist, Tsinghua University, Beijing 100084, China. (Email: hexiao@tsinghua.edu.cn)

Z. Wang is with the Department of Computer Science, Brunel University London, Uxbridge, Middlesex, UB8 3PH, United Kingdom. (Email: Zidong.Wangebrunel.ac.uk)

Q.-L. Han is with the School of Software and Electrical Engineering, Swinburne University of Technology, Melbourne, VIC 3122, Australia. (Email: qhaneswin.edu.au) on the MASs subject to disturbances [41], nonlinearities [5], [32], time-delays [33], noise [4], [8] as well as communication protocols [40].

In practical applications, actuator saturations serve as a ubiquitous phenomenon with examples including the motor speed restrained by the practical constraints and the deflection of the control surface for fixed-wing aircrafts limited by the physical structure [26]. Actuator saturations, if not appropriately dealt with, could lead to severe performance deterioration or even instability of system behaviors. In single-agent systems (SASs), there have been several techniques available in the literature that can deal with actuator saturations, for example, the low-gain feedback scheme [24], the anti-windup approach [1] and other methods in [39], [42]. Particularly, the low-gain feedback method has proven to be effective whose main idea is to employ a small gain so as to avoid the actuator saturation for any given initial states and, accordingly, the system can be simplified to a linear system with a slow convergence rate due to the small gain. To increase the convergence rate, the high-low-gain method has been proposed further in [25]. It is worth noting that, if the high gain is not chosen appropriately, the input variable may always switch between the upper and lower boundaries of the saturation, which is harmful to the system. In case that the global stability cannot be achieved, a compromise is to pursue the attractiveness by means of the socalled the domain of attraction (DOA) of a controller [15], see e.g. [16], [46], [52] on how to describe, estimate and enlarge the size of DOA.

In general, it is essentially difficult to apply the existing techniques for SASs to MASs in the presence of actuator saturations. The distinctive challenge stems from the topology of the MASs, that is, how to design a controller to compensate for the influence caused by the topology with limited execution capability. Note that the information coupling among agents poses an additional requirement on the consensus controller. So far, there have been some initial results handling the identified challenge, see e.g. [36], [37], where the leaderfollower semi-global state consensus and output consensus problems have been examined for a class of general linear MAS with actuator saturations. In addition, the global consensus problem has been investigated in [29] and [45]. An implicit assumption with almost all existing results is that the system is asymptotically null controllable with bounded controls. Such an assumption is rather stringent and one of the motivations of the current research is therefore to remove the constraints on the system matrices $A$ and $B$ by tackling more general linear 
This article has been accepted for publication in a future issue of this journal, but has not been fully edited. Content may change prior to final publication. Citation information: DOI 10.1109/TSMC.2021.3050370, IEEE Transactions on Systems, Man, and Cybernetics: Systems FINAL VERSION

MASs.

Intuitively, compared with SASs, MASs are more vulnerable to faults since MASs may suffer from faults from not only agents but also connections. As a result, the fault-tolerant control (FTC) problem for MASs arises whose aim is to maintain the desirable performance of each agent and also ensure the consensus of the MAS subject to fault occurrences. The existing FTC methods for MASs can be mainly categorized into passive FTC methods [7], [20], [50] and active FTC methods [6], [19]. For passive FTC problem, the controller is designed whose gain is large enough to tolerate all possible kinds of faults. For the active FTC problem, a unit of fault diagnosis is first exploited to detect, locate and identify the faults. Then, based on the obtained fault information, the controller is re-designed to guarantee the performance of the overall system.

It should be mentioned that traditional FTC methods cannot be directly applied to systems involving actuator saturations. The inherent difficulties resulting from the actuator saturations can be summarized as twofold. First, from the perspective of passive FTC methods, the limited execution capability of the actuator would largely reduce the fault-tolerant capability of the controller. Second, from the perspective of active FTC techniques, the saturation may lead to incorrect fault diagnosis results, incorrect compensation and even instability. In [34], a passive fault-tolerant controller is proposed for leader-follower MASs based on the robust controller. In this paper, we aim to look for control design algorithms with less conservatism by handling the following challenges: 1) how to eliminate the influence from the topology on the FTC problem? 2) how to design a low-conservative controller to deal with the degeneration of fault-tolerant capability caused by actuator saturations? and 3) how to optimize the controller parameters to find the largest DOA?

Stimulated by the discussion above, we address the faulttolerant consensus control problem for MASs with actuator saturations. The main contribution of this paper is the development of a novel saturation-resistant method for general linear MASs that include both the leaderless and leader-follower MASs. The saturation-resistant method consists of two steps, namely, the controller design step and the controller optimization step. More specially, in the step of controller design, the adaptive technique and the robust control method [44], [47], [53] are employed to guarantee the consensus. In the step of controller optimization, the concept of DOA is introduced for MASs and, through the use of dimension-reduction techniques, a novel optimization problem is formulated so as to find the largest DOA by solving several linear matrix inequalities (LMIs).

The remaining part of this paper is arranged as follows. In Section II, some preliminary background on communication graphs is introduced and the MAS model with actuator saturations/faults are formulated. In Section III, the design algorithm for the fault-tolerant controller is proposed, which is further optimized for the leaderless MAS. In Section IV, the fault-tolerant controller is investigated for the leader-follower MAS and subsequently optimized. In Section V, the results for numerical simulation are presented and, in Section VI, this paper is finally concluded.

Notations. Let $\mathbf{1}_{m}$ denote the $m \times 1$ column vector with all ones and $\mathbf{0}$ stand for the matrix with all zeros. $\lambda_{\min }(A)$ and $\lambda_{\max }(A)$ denote, respectively, the minimum and the maximum eigenvalues of the square matrix $A . \operatorname{diag}\left\{F_{0}, \ldots, F_{n}\right\}$ represents a block-diagonal matrix with $F_{0}, \ldots, F_{n}$ being its diagonal blocks. $\left[F_{i j}\right]_{m \times n}$ denotes a $m \times n$ matrix with elements $F_{i j} .|a|$ denotes the absolute value of a scalar $a$ and $\|x\|$ refers to the Euclidean norm of a vector $x$. The symbol $\otimes$ represents the Kronecker product.

\section{PROBLEM FORMULATION AND PRELIMINARIES}

\section{A. Preliminaries on graph theory}

For an MAS with $N$ agents, the communication between agents is described by a directed graph $\mathcal{G} \triangleq(\mathcal{V}, \mathcal{E}, \mathcal{A})$, where $\mathcal{V}=\left\{\mathcal{V}_{1}, \mathcal{V}_{2}, \ldots, \mathcal{V}_{N}\right\}$ represents the set of nodes, $\mathcal{E}$ is the set of edges, and $\mathcal{E}_{i j}=\left(\mathcal{V}_{i}, \mathcal{V}_{j}\right) \in \mathcal{E}$ if there is an information flow from node $\mathcal{V}_{i}$ to node $\mathcal{V}_{j}$. Let $N_{\mathcal{G}}$ denote the cardinality of $\mathcal{E}$, i.e. the number of edges. Note that $\mathcal{E}_{i j}$ and $\mathcal{E}_{j i}$ are counted as two edges. $\mathcal{A}=\left[a_{i j}\right]_{N \times N}$ is the adjacency matrix, where $a_{i j}=1$ if and only if $\mathcal{E}_{j i} \in \mathcal{E}$, otherwise $a_{i j}=0$. If $\mathcal{A}^{T}=\mathcal{A}$, then the graph is an undirected graph. The Laplacian matrix of the graph $\mathcal{G}$ (with $l_{i i}=\sum_{j \neq i} a_{i j}, l_{i j}=-a_{i j}, i \neq j$ ) is denoted as $L=\left[l_{i j}\right]_{N \times N}$. A directed graph $\mathcal{G}$ is said to contain a directed spanning tree if there exists a node that can reach any other nodes through paths. For undirected graphs, the existence of a directed spanning tree is equivalent to being connected.

If the undirected graph $\mathcal{G}$ is connected, then there exists an orthogonal matrix $T_{L}=\left[\begin{array}{ll}T_{0} & T_{1}\end{array}\right]$ such that

$$
T_{L}^{T} L T_{L}=\Lambda=\left[\begin{array}{llll}
0 & & & \\
& \lambda_{2} & & \\
& & \ddots & \\
& & & \lambda_{N}
\end{array}\right],
$$

where $T_{0}=\sqrt{\frac{1}{N}} \mathbf{1}_{N} \in \mathbb{R}^{N}, T_{1} \in \mathbb{R}^{N \times(N-1)}$ and $\lambda_{i}(i=$ $1,2, \ldots, N$ with $\lambda_{N} \geq \lambda_{N-1} \geq \ldots \geq \lambda_{2}>\lambda_{1}=0$ ) are the eigenvalues of $L$. Excluding the eigenvalue 0 , we define a positive definite matrix $\Phi=\operatorname{diag}\left\{\lambda_{2}, \ldots, \lambda_{N}\right\}$.

\section{B. MASs with actuator faults}

Consider the following MASs with $N$ agents subject to actuator saturations:

$$
\dot{x}_{i}=A x_{i}+B \sigma^{f}\left(u_{i}\right), \quad i \in \mathfrak{N} \triangleq\{1, \ldots, N\}
$$

where $x_{i} \in \mathbb{R}^{n}$ is the state variable and $u_{i} \in \mathbb{R}^{m}$ is the input variable, respectively. $\sigma^{f}\left(u_{i}\right) \triangleq\left[\sigma^{f}\left(u_{i 1}\right), \cdots, \sigma^{f}\left(u_{i m}\right)\right]^{T}$. $\sigma^{f}\left(u_{i}\right)$ denotes the imperfect actuators when faults meet saturations.

Consider the following loss-of-effectiveness fault and odd symmetric saturation:

$$
\left\{\begin{aligned}
\sigma^{f}\left(u_{i h}\right) & =\left(1-\rho_{i h}^{q}\right) \sigma\left(u_{i h}\right), 0 \leq \underline{\rho}_{i h}^{q} \leq \rho_{i h}^{q} \leq \bar{\rho}_{i h}^{q}<1, \\
\sigma\left(u_{i h}\right) & =\operatorname{sign}\left(u_{i h}\right) \min \left\{\left|u_{i h}\right|, 1\right\},
\end{aligned}\right.
$$


where $h \in \mathfrak{M} \triangleq\{1, \ldots, m\}, q \in \mathfrak{L} \triangleq\{1, \ldots, l\}$ and $i \in \mathfrak{N}$. Here, $q$ represents the $q$-th fault mode, and $\rho_{i h}^{q}$ stands for the unknown failure factor of the $h$-th actuator for the agent $i$, which is supposed to be invariant in the $q$-th fault mode. $\underline{\rho}_{i h}^{q}$ and $\bar{\rho}_{i h}^{q}$ are the lower and upper bounds of $\rho_{i h}^{q}$, respectively. Here, we exclude the condition of outage with $\rho_{i h}^{q}=1$.

Denoting $\sigma\left(u_{i}\right) \triangleq\left[\sigma\left(u_{i 1}\right), \cdots, \sigma\left(u_{i m}\right)\right]^{T}$ and $\rho_{i}^{q}=$ $\operatorname{diag}\left\{\rho_{i 1}^{q}, \ldots, \rho_{i m}^{q}\right\}$. For agent $i$, we have

$$
\dot{x}_{i}=A x_{i}+B\left(I-\rho_{i}^{q}\right) \sigma\left(u_{i}\right) .
$$

Letting $N_{\rho_{i}^{q}}$ be the set of upper/lower bounds of $\rho_{i h}^{q}$, it follows that

$$
N_{\rho_{i}^{q}}=\left\{\rho_{i}^{q} \mid \rho_{i}^{q}=\operatorname{diag}\left\{\rho_{i 1}^{q}, \ldots, \rho_{i m}^{q}\right\}, \rho_{i h}^{q} \in\left\{\underline{\rho}_{i h}^{q}, \bar{\rho}_{i h}^{q}\right\}\right\} .
$$

To obtain our main results, the following definitions and lemmas are needed.

Definition 1: (Domain of attraction [52]) A set is said to be inside the domain of attraction (DOA) if all the state trajectories starting from inside of it will remain in it. For $x(0)=x_{0}$, denote the state trajectory as $\psi\left(t, x_{0}\right)$. The DOA with respect to the origin is given by

$$
\mathbb{D}=\left\{x_{0} \mid \lim _{t \rightarrow \infty} \psi\left(t, x_{0}\right)=0\right\} .
$$

Definition 2: (Leaderless Consensus [23]) The consensus is reached asymptotically by a distributed controller if

$$
\lim _{t \rightarrow \infty}\left\|x_{i}(t)-x_{j}(t)\right\|=0
$$

for $i, j \in \mathfrak{N}$.

Definition 3: (Leader-follower Consensus [23]) The leaderfollower consensus is reached asymptotically by a distributed controller if

$$
\lim _{t \rightarrow \infty}\left\|x_{i}(t)-x_{1}(t)\right\|=0
$$

for $i \in \mathcal{N} \triangleq\{2, \ldots, N\}$.

Lemma 1: [13] For matrices $A, B, C$ and $D$, the Kronecker product $\otimes$ has the following properties:

(1) $(A+B) \otimes C=A \otimes C+B \otimes C$,

(2) $(A \otimes B)(C \otimes D)=(A C) \otimes(B D)$,

(3) $(A \otimes B)^{T}=A^{T} \otimes B^{T}$.

Lemma 2: Suppose that the undirected graph $\mathcal{G}$ is connected. If there exist matrices $P_{1} \geq 0$ and $P_{2} \leq 0$, then

$$
\begin{gathered}
L^{2} \otimes P_{1} \geq \lambda_{2} L \otimes P_{1} \\
L^{2} \otimes P_{2} \geq \lambda_{N} L \otimes P_{2} .
\end{gathered}
$$

Proof: It follows readily from the facts $L=T_{1} \Phi T_{1}^{T}$ and $T_{1}^{T} T_{1}=I_{N-1}$ that

$$
\begin{aligned}
L^{2} \otimes P_{1}-\lambda_{2} L \otimes P_{1} & =\left(T_{1} \Phi^{2} T_{1}^{T}-\lambda_{2} T_{1} \Phi T_{1}^{T}\right) \otimes P_{1} \\
& =\left(T_{1}\left(\Phi^{2}-\lambda_{2} \Phi\right) T_{1}^{T}\right) \otimes P_{1} \\
& \geq 0
\end{aligned}
$$

and

$$
L^{2} \otimes P_{2}-\lambda_{N} L \otimes P_{2}=\left(L^{2}-\lambda_{N} L\right) \otimes P_{2} \geq 0 .
$$

The proof is complete.
Lemma 3: [44] Define a matrix $\Theta$ as

$$
\Theta=\left[\begin{array}{cc}
\Theta_{11} & \Theta_{12} \\
\Theta_{12}^{T} & \Theta_{22}
\end{array}\right],
$$

where $\Theta_{22}=\operatorname{diag}\left\{\Theta_{22}^{1}, \ldots, \Theta_{22}^{p}, \ldots, \Theta_{22}^{m}\right\} \quad(p \in \mathfrak{M})$ with $\Theta_{22}^{p} \in \mathbb{R}^{n \times n}$ and $\Theta_{11} \in \mathbb{R}^{m n \times m n}$. If there exists $\Theta$ such that the following inequalities hold

$$
\begin{gathered}
\Theta_{22}^{p} \leq 0, \\
\Theta_{11}+\Theta_{12} \Delta(\delta)+\left(\Theta_{12} \Delta(\delta)\right)^{T}+\Delta(\delta) \Theta_{22} \Delta(\delta) \geq 0, \\
{\left[\begin{array}{cc}
Q & E \\
E^{T} & F
\end{array}\right]+G^{T} \Theta G<0,}
\end{gathered}
$$

then the inequality

$$
\begin{aligned}
W(\delta)= & Q+\sum_{j=1}^{m} \delta_{j} E_{j}+\left(\sum_{j=1}^{m} \delta_{j} E_{j}\right)^{T} \\
& +\sum_{j=1}^{m} \sum_{k=1}^{m} \delta_{j} \delta_{k} F_{j k}<0
\end{aligned}
$$

holds for all possible $\delta_{j} \in\left[\underline{\delta}_{j}, \bar{\delta}_{j}\right]$, where $Q=Q^{T} \in \mathbb{R}^{n \times n}$ and $F_{j k}=F_{j k}^{T} \in \mathbb{R}^{n \times n}, E_{j} \in \mathbb{R}^{n \times n}, \delta \in \Delta_{\delta}$,

$$
\begin{gathered}
E=\left[E_{1}, E_{2}, \cdots, E_{m}\right], F=\left[F_{j k}\right]_{m n \times m n}, \\
\Delta_{\delta}=\left\{\delta=\left[\delta_{1}, \cdots, \delta_{m}\right]: \delta_{j} \in\left\{\underline{\delta}_{j}, \bar{\delta}_{j}\right\}\right\}, \\
\Delta(\delta)=\operatorname{diag}\left\{\delta_{1} I_{n}, \ldots, \delta_{m} I_{n}\right\}, \\
G=\left[\begin{array}{cc}
\mathbf{1}_{m} \otimes I_{n} & \mathbf{0} \\
\mathbf{0} & I_{m n}
\end{array}\right] .
\end{gathered}
$$

\section{LEADERLESS CONSENSUS}

In this section, we consider the FTC problem for leaderless MASs. The saturation-resistant controller design consists of two steps. The first step is to determine the controller form and give a sufficient condition under which the leaderless consensus can be reached. The second step is to optimize the parameters of the controller by solving a set of LMIs to obtain the largest DOA.

\section{A. Consensus controller}

Considering the MAS with each agent described by (2), we design the controller as follows:

$$
\begin{aligned}
u_{i}= & K\left(\hat{\rho}_{i}\right) \sum_{j=1}^{N} a_{i j}\left(x_{i}-x_{j}\right) \\
& -c B^{T} P^{-1} \sum_{j=1}^{N} a_{i j}\left(x_{i}-x_{j}\right)
\end{aligned}
$$

where

$$
\begin{aligned}
& K\left(\hat{\rho}_{i}\right)=K_{0}+K_{a}\left(\hat{\rho}_{i}\right)+K_{b}\left(\hat{\rho}_{i}\right), \\
& K_{a}\left(\hat{\rho}_{i}\right)=\sum_{h=1}^{m} K_{a h} \hat{\rho}_{i h}, K_{b}\left(\hat{\rho}_{i}\right)=\sum_{h=1}^{m} K_{b h} \hat{\rho}_{i h} .
\end{aligned}
$$

Here, $\hat{\rho}_{i}=\operatorname{diag}\left\{\hat{\rho}_{i 1}, \ldots, \hat{\rho}_{i m}\right\}$ is the adaptive parameter, and $c, K_{0}, K_{a h}$ and $K_{b h}$ are feedback gains to be designed. 
Define $\tilde{\rho}_{i}=\operatorname{diag}\left\{\tilde{\rho}_{i 1}, \ldots, \tilde{\rho}_{i m}\right\}$ and $\tilde{\rho}_{i h}=\hat{\rho}_{i h}-\rho_{i h}^{q}$. Then, $\left(I-\rho_{i}^{q}\right) K\left(\hat{\rho}_{i}\right)$ can be rewritten as

$$
\begin{aligned}
\left(I-\rho_{i}^{q}\right) K\left(\hat{\rho}_{i}\right)= & \left(I-\rho_{i}^{q}\right) K_{0}+K_{a}\left(\rho_{i}^{q}\right)-\rho_{i}^{q} K_{a}\left(\hat{\rho}_{i}\right) \\
& +\left(I-\hat{\rho}_{i}\right) K_{b}\left(\hat{\rho}_{i}\right)+K_{a}\left(\tilde{\rho}_{i}\right) \\
& +\tilde{\rho}_{i} K_{b}\left(\hat{\rho}_{i}\right) .
\end{aligned}
$$

Before moving on, we define the following matrices:

$$
\begin{aligned}
M & \triangleq \operatorname{diag}\left\{B\left(I-\rho_{1}^{q}\right), \ldots, B\left(I-\rho_{N}^{q}\right)\right\} \\
S & \triangleq \operatorname{diag}\left\{K\left(\hat{\rho}_{1}\right), \ldots, K\left(\hat{\rho}_{N}\right)\right\} \\
H_{0} & \triangleq S-c I_{N} \otimes B^{T} P^{-1} \\
H & \triangleq H_{0}\left(T_{1} \Phi \otimes I_{n}\right) .
\end{aligned}
$$

Next, we transform the consensus problem of the MAS into a stability problem of a multi-input multi-output system through the following three steps.

s1) By denoting $x \triangleq\left[x_{1}^{T}, x_{2}^{T}, \cdots, x_{N}^{T}\right]^{T}$, the collective dynamics of the MAS can then be rewritten as:

$$
\dot{x}=\left(I_{N} \otimes A\right) x+M \sigma\left(H_{0}\left(L \otimes I_{n}\right) x\right) .
$$

s2) Letting $y=\left(T_{L}^{T} \otimes I_{n}\right) x \triangleq\left[y_{1}^{T}, y_{2}^{T}, \cdots, y_{N}^{T}\right]^{T}$ where $y_{i} \in \mathbb{R}^{n}(i \in \mathfrak{N})$, we further convert (6) into

$$
\dot{y}=\left(I_{N} \otimes A\right) y+\left(T_{L}^{T} \otimes I_{n}\right) M \sigma\left(H_{0}\left(L T_{L} \otimes I_{n}\right) y\right) .
$$

s3) Finally, denoting $z \triangleq\left[y_{2}^{T}, \cdots, y_{N}^{T}\right]^{T}=\left(T_{1}^{T} \otimes I_{n}\right) x$, we obtain

$$
\dot{z}=\left(I_{N-1} \otimes A\right) z+\left(T_{1}^{T} \otimes I_{n}\right) M \sigma(H z) .
$$

For a matrix $Q$, define

$$
\wp(Q)=\left\{z \in \mathbb{R}^{n(N-1)}:\left|Q_{j} z\right| \leq 1, \forall j\right\},
$$

where $Q_{j}$ represents the $j$-th row of the matrix $Q$.

Let $P$ be a positive definite matrix. For $t_{0}>0(i \in \mathfrak{N}, h \in$ $\mathfrak{M})$, we denote

$$
\begin{gathered}
\varepsilon\left(\Phi \otimes P^{-1}, 1\right)=\left\{z \in \mathbb{R}^{n(N-1)}: \frac{1}{2} z^{T}\left(\Phi \otimes P^{-1}\right) z \leq 1\right\}, \\
\varepsilon^{*}\left(\Phi \otimes P^{-1}, 1\right)=\left\{z \in \mathbb{R}^{n(N-1)}: \frac{1}{2} z^{T}\left(\Phi \otimes P^{-1}\right) z\right. \\
\left.\quad+\sum_{i=1}^{N} \sum_{h=1}^{m} \frac{\tilde{\rho}_{i h}^{2}}{2 t_{0}} \leq 1\right\} .
\end{gathered}
$$

Theorem 1: Let the undirected graph $\mathcal{G}$ be connected. Leaderless consensus of agents modeled by (2) is achieved by the controller (3) within the set $\varepsilon^{*}\left(\Phi \otimes P^{-1}, 1\right)$ if there exist a constant $k>0$ and matrices $P>0, Q, E, F, G, Y_{0}, Y_{a h}, Y_{b h}$,

$$
\Theta^{i}=\left[\begin{array}{cc}
\Theta_{11}^{i} & \Theta_{12}^{i} \\
\left(\Theta_{12}^{i}\right)^{T} & \Theta_{22}^{i}
\end{array}\right]
$$

with $\Theta_{22}^{i}=\operatorname{diag}\left\{\Theta_{22}^{i 1}, \ldots, \Theta_{22}^{i m}\right\}, \Theta_{11}^{i} \in \mathbb{R}^{m n \times m n}, \Theta_{22}^{i p} \in$ $\mathbb{R}^{n \times n}, \quad \Theta_{22}^{i p} \leq 0(h, p \in \mathfrak{M}, i \in \mathfrak{N})$ satisfying $\varepsilon^{*}(\Phi \otimes$ $\left.P^{-1}, 1\right) \subset \wp(H)$ and the following conditions:

$$
c\left(1-\max _{i, h, q}\left\{\bar{\rho}_{i h}^{q}\right\}\right) \geq \frac{1}{k \lambda_{2}},
$$

$$
\begin{gathered}
A P+P A^{T}-\frac{2}{k} B B^{T}<0 \\
\Theta_{11}^{i}+\Theta_{12}^{i} \Delta\left(\hat{\rho}_{i}\right)+\left(\Theta_{12}^{i} \Delta\left(\hat{\rho}_{i}\right)\right)^{T}+\Delta\left(\hat{\rho}_{i}\right) \Theta_{22}^{i} \Delta\left(\hat{\rho}_{i}\right) \geq 0 \\
{\left[\begin{array}{cc}
Q & E \\
E^{T} & F
\end{array}\right]+G^{T} \Theta^{i} G<0}
\end{gathered}
$$

where $\rho_{i}=\operatorname{diag}\left\{\rho_{i 1}, \ldots, \rho_{i m}\right\} \in\left\{\rho_{i}^{1}, \ldots, \rho_{i}^{l}\right\}, \rho_{i}^{q} \in N_{\rho_{i}^{q}}$, $q \in \mathfrak{L}, \hat{\rho}_{i} \in \Delta_{\hat{\rho}_{i}}, \Delta\left(\hat{\rho}_{i}\right)=\operatorname{diag}\left\{\hat{\rho}_{i 1} I_{n}, \ldots, \hat{\rho}_{i m} I_{n}\right\}$ and

$$
\begin{aligned}
\Delta_{\hat{\rho}_{i}}=\left\{\hat{\rho}_{i}=\left[\hat{\rho}_{i 1}, \cdots, \hat{\rho}_{i m}\right]: \hat{\rho}_{i h} \in\left\{\min _{q}\left\{\underline{\rho}_{i h}^{q}\right\},\right.\right. \\
\left.\left.\max _{q}\left\{\hat{\rho}_{i h}^{q}\right\}\right\}\right\}, \\
Q=\frac{1}{\lambda_{N}} A P+\left(\frac{1}{\lambda_{N}} A P\right)^{T}+B\left(I-\rho_{i}\right) Y_{0} \\
+\left(B\left(I-\rho_{i}\right) Y_{0}\right)^{T}+B \sum_{h=1}^{m} \rho_{i h} Y_{a h} \\
+\left(B \sum_{h=1}^{m} \rho_{i h} Y_{a h}\right)^{T}-\frac{2}{k \lambda_{N}} B B^{T}, \\
E=-B \rho_{i} Y_{a}+B Y_{b},
\end{aligned}
$$

$$
F=\left[\begin{array}{c}
-B^{1} \\
\vdots \\
-B^{m}
\end{array}\right] Y_{b}+\left(\left[\begin{array}{c}
-B^{1} \\
\vdots \\
-B^{m}
\end{array}\right] Y_{b}\right)^{T}
$$

$$
\begin{gathered}
B=\left[b^{1}, b^{2}, \cdots, b^{m}\right], B^{h}=\left[0, \cdots, b^{h}, \cdots\right], \\
Y_{a}=\left[Y_{a 1}, Y_{a 1}, \cdots, Y_{a m}\right], Y_{b}=\left[Y_{b 1}, Y_{b 1}, \cdots, Y_{b m}\right], \\
G=\left[\begin{array}{cc}
\mathbf{1}_{m} \otimes I_{n} & \mathbf{0} \\
\mathbf{0} & I_{m n}
\end{array}\right] .
\end{gathered}
$$

In addition, the feedback gains can be designed as

$$
K_{0}=Y_{0} P^{-1}, K_{a h}=Y_{a h} P^{-1}, K_{b h}=Y_{b h} P^{-1},
$$

and $\hat{\rho}_{i h}$ is determined according to the following adaptive law

$$
\dot{\hat{\rho}}_{i h}= \begin{cases}0, & \text { if } \hat{\rho}_{i h}=\min _{q}\left\{\underline{\rho}_{i h}^{q}\right\} \text { and } T_{i h} \leq 0 \\ & \text { or } \hat{\rho}_{i h}=\max _{q}\left\{\bar{\rho}_{i h}^{q}\right\} \text { and } T_{i h} \geq 0 \\ T_{i h}, & \text { otherwise }\end{cases}
$$

with

$$
\begin{aligned}
T_{i h}= & -t_{0}\left(\sum_{j=1}^{N} a_{i j}\left(x_{i}-x_{j}\right)\right)^{T}\left(P^{-1} B^{h} K_{b}\left(\hat{\rho}_{i}\right)\right. \\
& \left.+P^{-1} B K_{a h}\right)\left(\sum_{j=1}^{N} a_{i j}\left(x_{i}-x_{j}\right)\right), t_{0}>0 .
\end{aligned}
$$

Proof: If $z \in \wp(H)$, that is, $\left|H_{j} z\right| \leq 1$, then the system (8) can be simplified as

$$
\dot{z}=\left(I_{N-1} \otimes A\right) z+\left(T_{1}^{T} \otimes I_{n}\right) M H_{0}\left(T_{1} \Phi \otimes I_{n}\right) z .
$$


Before giving the Lyapunov function, we first denote that

$$
\begin{aligned}
\Psi \triangleq & \operatorname{diag}\left\{B\left(I-\rho_{1}^{q}\right) K\left(\hat{\rho}_{1}\right), \ldots, B\left(I-\rho_{N}^{q}\right) K\left(\hat{\rho}_{N}\right)\right\} \\
\Gamma \triangleq & \operatorname{diag}\left\{\Gamma_{1}, \ldots, \Gamma_{N}\right\}, \Lambda \triangleq \operatorname{diag}\left\{\Lambda_{1}, \ldots, \Lambda_{N}\right\} \\
\Gamma_{i} \triangleq & P^{-1} B\left(\left(I-\rho_{i}^{q}\right) K_{0}+K_{a}\left(\rho_{i}^{q}\right)-\rho_{i}^{q} K_{a}\left(\hat{\rho}_{i}\right)\right. \\
& \left.+\left(I-\hat{\rho}_{i}\right) K_{b}\left(\hat{\rho}_{i}\right)\right), \\
\Lambda_{i} \triangleq & \frac{1}{\lambda_{N}}\left(P^{-1} A+A^{T} P^{-1}-2 / k P^{-1} B B^{T} P^{-1}\right) \\
& +\Gamma_{i}+\Gamma_{i}^{T} .
\end{aligned}
$$

Choose the following Lyapunov function

$$
V=\frac{1}{2} z^{T}\left(\Phi \otimes P^{-1}\right) z+\sum_{i=1}^{N} \sum_{h=1}^{m} \frac{\tilde{\rho}_{i h}^{2}}{2 t_{0}}
$$

whose time derivative is evaluated as

$$
\begin{aligned}
\dot{V}= & z^{T}\left(\Phi \otimes P^{-1}\right) \dot{z}+\sum_{i=1}^{N} \sum_{h=1}^{m} \frac{\tilde{\rho}_{i h} \dot{\hat{\rho}}_{i h}}{t_{0}} \\
= & z^{T}\left(\Phi \otimes P^{-1} A\right) z+\sum_{i=1}^{N} \sum_{h=1}^{m} \frac{\tilde{\rho}_{i h} \dot{\hat{\rho}}_{i h}}{t_{0}} \\
& +z^{T}\left(\Phi T_{1}^{T} \otimes I_{n}\right) M H_{0}\left(T_{1} \Phi \otimes I_{n}\right) z .
\end{aligned}
$$

Substituting $z=\left(T_{1}^{T} \otimes I_{n}\right) x$ into (15) yields

$$
\begin{aligned}
& \dot{V}=x^{T}\left(L \otimes P^{-1} A\right) x+\sum_{i=1}^{N} \sum_{h=1}^{m} \frac{\tilde{\rho}_{i h} \dot{\hat{\rho}}_{i h}}{t_{0}} \\
& +x^{T}\left(L \otimes P^{-1}\right) M H_{0}\left(L \otimes I_{n}\right) x \\
& =x^{T}\left(L \otimes P^{-1} A\right) x+\sum_{i=1}^{N} \sum_{h=1}^{m} \frac{\tilde{\rho}_{i h} \dot{\hat{\rho}}_{i h}}{t_{0}} \\
& -c x^{T}\left(L \otimes P^{-1}\right) M\left(L \otimes B^{T} P^{-1}\right) x \\
& +x^{T}\left(L \otimes I_{n}\right)\left(I_{N} \otimes P^{-1}\right) \Psi\left(L \otimes I_{n}\right) x \\
& =x^{T}\left(L \otimes P^{-1} A\right) x+\sum_{i=1}^{N} \sum_{h=1}^{m} \frac{\tilde{\rho}_{i h} \dot{\hat{\rho}}_{i h}}{t_{0}} \\
& -c x^{T}\left(L \otimes P^{-1}\right) M\left(L \otimes B^{T} P^{-1}\right) x \\
& +\left[\begin{array}{c}
\sum_{j=1}^{N} l_{1 j} x_{j} \\
\vdots \\
\sum_{j=1}^{N} l_{N j} x_{j}
\end{array}\right]^{T}\left(I_{N} \otimes P^{-1}\right) \Psi\left[\begin{array}{c}
\sum_{j=1}^{N} l_{1 j} x_{j} \\
\vdots \\
\sum_{j=1}^{N} l_{N j} x_{j}
\end{array}\right] \\
& =x^{T}\left(L \otimes P^{-1} A\right) x+\sum_{i=1}^{N} \sum_{h=1}^{m} \frac{\tilde{\rho}_{i h} \dot{\hat{\rho}}_{i h}}{t_{0}} \\
& -c x^{T}\left(L \otimes P^{-1}\right) M\left(L \otimes B^{T} P^{-1}\right) x \\
& +\left(\sum_{j=1}^{N} l_{1 j} x_{j}\right)^{T} P^{-1} B\left(I-\rho_{1}^{q}\right) K\left(\hat{\rho}_{1}\right) \sum_{j=1}^{N} l_{1 j} x_{j} \\
& +\ldots \\
& +\left(\sum_{j=1}^{N} l_{N j} x_{j}\right)^{T} P^{-1} B\left(I-\rho_{N}^{q}\right) K\left(\hat{\rho}_{N}\right) \sum_{j=1}^{N} l_{N j} x_{j} .
\end{aligned}
$$

Noting that

$$
\begin{aligned}
P^{-1} B \tilde{\rho} K_{b}\left(\hat{\rho}_{i}\right) & =\sum_{h=1}^{m} \tilde{\rho}_{i h} P^{-1} B^{h} K_{b}\left(\hat{\rho}_{i}\right) \\
P^{-1} B K_{a}\left(\tilde{\rho}_{i}\right) & =\sum_{h=1}^{m} \tilde{\rho}_{i h} P^{-1} B K_{a h},
\end{aligned}
$$

together with (5) and the adaptive law, we obtain that

$$
\begin{aligned}
\dot{V} \leq & x^{T}\left(L \otimes P^{-1} A\right) x \\
& +\left(\sum_{j=1}^{N} l_{1 j} x_{j}\right)^{T} \Gamma_{1} \sum_{j=1}^{N} l_{1 j} x_{j}+\ldots \\
& +\left(\sum_{j=1}^{N} l_{N j} x_{j}\right)^{T} \Gamma_{N} \sum_{j=1}^{N} l_{N j} x_{j} \\
& -c x^{T}\left(L \otimes P^{-1}\right) M\left(L \otimes B^{T} P^{-1}\right) x \\
= & x^{T}\left(L \otimes P^{-1} A\right) x+x^{T}\left(L \otimes I_{n}\right) \Gamma\left(L \otimes I_{n}\right) x \\
& -c x^{T}\left(L \otimes P^{-1}\right) M\left(L \otimes B^{T} P^{-1}\right) x .
\end{aligned}
$$

On the other hand, we have

$$
\begin{aligned}
& -c x^{T}\left(L \otimes P^{-1}\right) M\left(L \otimes B^{T} P^{-1}\right) x \\
=- & c x^{T}\left(L \otimes P^{-1} B\right)\left[\begin{array}{ccc}
I-\rho_{1}^{q} & & \\
& \ddots & \\
& & I-\rho_{N}^{q}
\end{array}\right] \\
& \times\left(L \otimes B^{T} P^{-1}\right) x \\
\leq & -c\left(1-\max _{i, h, q}\left\{\bar{\rho}_{i h}^{q}\right\}\right) x^{T}\left(L^{2} \otimes P^{-1} B B^{T} P^{-1}\right) x \\
\leq & -c \lambda_{2}\left(1-\max _{i, h, q}\left\{\tilde{\rho}_{i h}^{q}\right\}\right) x^{T}\left(L \otimes P^{-1} B B^{T} P^{-1}\right) x \\
\leq & -\frac{1}{k} x^{T}\left(L \otimes P^{-1} B B^{T} P^{-1}\right) x .
\end{aligned}
$$

It follows that

$$
\begin{aligned}
\dot{V} \leq & x^{T}\left(L \otimes P^{-1} A\right) x+x^{T}\left(L \otimes I_{n}\right) \Gamma\left(L \otimes I_{n}\right) x \\
& -\frac{1}{k} x^{T}\left(L \otimes P^{-1} B B^{T} P^{-1}\right) x \\
= & \frac{1}{2} x^{T}\left(L \otimes \left(-2 / k P^{-1} B B^{T} P^{-1}+P^{-1} A\right.\right. \\
& \left.\left.+A^{T} P^{-1}\right)\right) x+x^{T}\left(L \otimes I_{n}\right) \Gamma\left(L \otimes I_{n}\right) x .
\end{aligned}
$$

According to (9b) and Lemma 2, we have

$$
\begin{aligned}
\dot{V} & \leq \frac{1}{2} x^{T}\left(L \otimes I_{n}\right) \Lambda\left(L \otimes I_{n}\right) x \\
& =z^{T}\left(\Phi \otimes I_{n}\right)\left(T_{1}^{T} \otimes I_{n}\right) \Lambda\left(T_{1} \otimes I_{n}\right)\left(\Phi \otimes I_{n}\right) z .
\end{aligned}
$$

By virtue of Lemma 3, if conditions (9a)-(9d) hold, then $\Lambda_{i}<0$. Noting that $T_{1}$ is column full rank, we can thus conclude from the LaSalle's Invariance principle [18] that $\lim _{t \rightarrow \infty} z=\left[y_{2}^{T}, \cdots, y_{N}^{T}\right]^{T}=\mathbf{0}$.

Consequently, we have

$$
\begin{aligned}
x & =\left(T_{L} \otimes I_{n}\right) y=\left[\begin{array}{ll}
T_{0} \otimes I_{n} & T_{1} \otimes I_{n}
\end{array}\right] y \\
& =\left(T_{0} \otimes I_{n}\right) y_{1}=\left(\sqrt{\frac{1}{N}} \mathbf{1}_{N} \otimes I_{n}\right) y_{1},
\end{aligned}
$$


This article has been accepted for publication in a future issue of this journal, but has not been fully edited. Content may change prior to final publication. Citation information: DOI 10.1109/TSMC.2021.3050370, IEEE Transactions on Systems, Man, and Cybernetics: Systems

which concludes that $N$ agents can ultimately reach consensus within $\varepsilon^{*}\left(\Phi \otimes P^{-1}, 1\right)$. By recalling that $\varepsilon^{*}\left(\Phi \otimes P^{-1}, 1\right) \subset$ $\wp(H)$, the proof of Theorem 1 ends here.

\section{B. Domain of attraction}

Recall from Theorem 1 that the condition $\varepsilon^{*}\left(\Phi \otimes P^{-1}, 1\right) \subset$ $\wp(H)$ holds if $\varepsilon\left(\Phi \otimes P^{-1}, 1\right) \subset \wp(H)$ holds. Combining

$$
\varepsilon\left(\Phi \otimes P^{-1}, 1\right) \subset \varepsilon\left(\frac{1}{\lambda_{N}} \Phi^{2} \otimes P^{-1}, 1\right)
$$

and

$$
\Phi^{2} \otimes P^{-1}=\left(\Phi T_{1}^{T} \otimes I_{n}\right)\left(I_{N} \otimes P^{-1}\right)\left(T_{1} \Phi \otimes I_{n}\right),
$$

we know that $\varepsilon\left(\Phi \otimes P^{-1}, 1\right) \subset \wp(H)$ holds if

$$
\varepsilon\left(\frac{1}{\lambda_{N}} I_{N} \otimes P^{-1}, 1\right) \subset \wp\left(H_{0}\right)
$$

holds. Furthermore, note that $\varepsilon\left(\frac{1}{\lambda_{N}} I_{N} \otimes P^{-1}, 1\right) \subset \wp\left(H_{0}\right)$ if and only if the following inequality holds for all $j=$ $1, \ldots, m N$ :

$$
\left[\begin{array}{cc}
\frac{1}{2 \lambda_{N}} & h_{j}\left(I_{N} \otimes P\right) \\
* & I_{N} \otimes P
\end{array}\right] \geq 0
$$

where $h_{j}$ is the $j$-th row of $H_{0}$. Moreover, we have

$$
\begin{aligned}
& H_{0}\left(I_{N} \otimes P\right) \\
& =\left[\begin{array}{lll}
K\left(\hat{\rho}_{1}\right) P-c B^{T} & \\
& \ddots & \\
& & K\left(\hat{\rho}_{N}\right) P-c B^{T}
\end{array}\right] .
\end{aligned}
$$

To this end, by the Schur Complement Lemma, inequality (21) holds if and only if

$$
\left[\begin{array}{cc}
\frac{1}{2 \lambda_{N}} & \left(K\left(\hat{\rho}_{i}\right) P-c B^{T}\right)_{j} \\
* & P
\end{array}\right] \geq 0
$$

holds for all $i=1, \ldots, N$ and the index $j(j=1, \ldots, m)$ denotes the $j$-th row of $K\left(\hat{\rho}_{i}\right) P-c B^{T}$.

Based on (10), inequality (23) can be further expressed as

$$
\begin{aligned}
& {\left[\begin{array}{cc}
\frac{-1}{2 \lambda_{N}} & -\left(Y_{0}-c B^{T}\right)_{j} \\
* & -P
\end{array}\right]} \\
& +\sum_{h=1}^{m} \hat{\rho}_{i h}\left[\begin{array}{cc}
0 & -\left(Y_{a h}+Y_{b h}\right)_{j} \\
* & \mathbf{0}
\end{array}\right] \leq 0
\end{aligned}
$$

for all $\hat{\rho}_{i} \in \Delta_{\hat{\rho}_{i}}$.

In order to seek the largest DOA of the proposed controller, we introduce a prescribed ellipsoid $\epsilon(\Phi \otimes R, 1)=\{z \in$ $\left.\mathbb{R}^{n(N-1)}: z^{T}(\Phi \otimes R) z \leq 1\right\}$ and use $\max \alpha$ to estimate the DOA of the controller, where $\alpha$ satisfies

$$
\alpha \epsilon(\Phi \otimes R, 1) \subset \varepsilon\left(\Phi \otimes P^{-1}, 1\right) .
$$

Finally, we arrive at the following optimization problem:

$$
\hat{\max }_{i}, \rho_{i}, k, P, Y_{0}, Y_{a}, Y_{b} \quad \alpha, \quad \text { s.t. (9), (24), (25), }
$$

which can be rewritten as

$$
\min _{\hat{\rho}_{i}, \rho_{i}, k, P, Y_{0}, Y_{a}, Y_{b}} \gamma \text {, s.t. (9), (24), } R^{-1} \leq 2 \gamma P .
$$

where $\gamma=\alpha^{-2}$.

Remark 1: Based on the above analysis, the initial state $x(0)$ satisfying $z(0)=\left(T_{1}^{T} \otimes I_{n}\right) x(0) \in \varepsilon\left(\Phi \otimes P^{-1}, 1\right)$ is contained in the DOA. Furthermore,

$$
\begin{aligned}
& z(0)^{T}\left(\Phi \otimes P^{-1}\right) z(0) \\
& =x(0)^{T}\left(L \otimes P^{-1}\right) x(0) \\
& \leq \lambda_{\min }^{-1}(P) x(0)^{T}\left(L \otimes I_{n}\right) x(0) \\
& =\frac{1}{\lambda_{\min }(P)} \sum_{\mathcal{E}_{i j} \in \mathcal{E}, i<j}\left\|x_{i}(0)-x_{j}(0)\right\|^{2} \\
& \leq \frac{N_{\mathcal{G}}}{2 \lambda_{\min }(P)} \max _{i, j, \mathcal{E}_{i j} \in \mathcal{E}}\left\{\left\|x_{i}(0)-x_{j}(0)\right\|^{2}\right\} .
\end{aligned}
$$

It follows that the initial state $x_{i}(0)$ of each agent satisfying

$$
\max _{i, j, \mathcal{E}_{i j} \in \mathcal{E}}\left\{\left\|x_{i}(0)-x_{j}(0)\right\|\right\} \leq 2 \sqrt{\frac{\lambda_{\min }(P)}{N_{\mathcal{G}}}}
$$

is contained in the DOA, which means that the consensus of agents can be achieved by the controller (3) with parameters designed by (27) for any $x_{i}(0)$ satisfying (28).

Remark 2: In Theorem 1, if we set $Y_{a h}=\mathbf{0}, Y_{b h}=\mathbf{0}$ and $\Theta^{i}=\mathbf{0}$, then the conditions of Theorem 1 can be reduced to (9a), (9b) and

$$
\begin{aligned}
Q= & \frac{1}{\lambda_{N}}\left(A P+P A^{T}-\frac{2}{k} B B^{T}\right) \\
& +B\left(I-\rho_{i}\right) Y_{0}+\left(B\left(I-\rho_{i}\right) Y_{0}\right)^{T}<0 .
\end{aligned}
$$

The corresponding controller is

$$
u_{i}=\left(K_{0}-c B^{T} P^{-1}\right) \sum_{j=1}^{N} a_{i j}\left(x_{i}-x_{j}\right),
$$

which is the traditional fault-tolerant controller with fixed gain$\mathrm{s}$ [11]. The above analysis shows that the results concerning the fixed gain controller can be seen as a special case of Theorem 1. Therefore, the proposed fault-tolerant controller in Theorem 1 is more general than the fixed-gain controller. In addition, the adaptive mechanism of the proposed controller in Theorem 1 helps to achieve better dynamic performance, which will be illustrated in the simulation part.

\section{LEADER-FOLLOWER CONSENSUS}

In some cases, we want the agents to track a desired trajectory, and this gives rise to the leader-follower consensus problem. In this section, we aim to tackle the leader-follower consensus problem where only partial followers can receive the leader's state information. The agent indexed by 1 is the leader and agents indexed by $2, \ldots, N$ are followers. To start with, the following assumptions are presented to facilitate our analysis.

Assumption 1: The graph $\mathcal{G}$ contains a directed spanning tree with the leader as the root, and the subgraph associated with the followers is an undirected graph.

Assumption 2: The input of the leader is bounded, i.e $\left\|u_{1}\right\| \leq \gamma_{1}$, where $0<\gamma_{1} \leq 1$. 
Let the Laplacian matrix of graph $\mathcal{G}$ be denoted by $L \in$ $\mathbb{R}^{n \times n}$, which can be partitioned as

$$
\left[\begin{array}{cc}
0 & \mathbf{0}_{1 \times(N-1)} \\
L_{2} & L_{1}
\end{array}\right]
$$

with $L_{1}>0$ [23].

\section{A. Consensus controller}

Based on the results obtained in the leaderless consensus case, the controller of the follower $i(i \in \mathcal{N})$ is designed as

$$
\begin{aligned}
u_{i}= & K\left(\hat{\rho}_{i}\right) \sum_{j=1}^{N} a_{i j}\left(x_{i}-x_{j}\right) \\
& +c \pi\left(-B^{T} P^{-1} \sum_{j=1}^{N} a_{i j}\left(x_{i}-x_{j}\right)\right),
\end{aligned}
$$

where $K\left(\hat{\rho}_{i}\right)$ is designed in the same way of (4). $c, K_{0}, K_{a h}$ and $K_{b h}$ are feedback gains to be designed later. Given a vector $\omega=\left[\omega_{1}, \omega_{2}, \cdots, \omega_{m}\right]^{T}$, the nonlinear function $\pi(\omega)$ is defined as $\pi(\omega)=\left[\pi\left(\omega_{1}\right), \pi\left(\omega_{2}\right), \cdots, \pi\left(\omega_{m}\right)\right]^{T}$ with

$$
\pi\left(\omega_{t}\right)=\left\{\begin{array}{ll}
\omega_{t}+\operatorname{sign}\left(\omega_{t}\right), & \omega_{t} \neq 0 \\
0, & \omega_{t}=0
\end{array} .\right.
$$

Let $e_{i}=x_{i}-x_{1}, i \in \mathcal{N}$ and $e=\left[e_{2}^{T}, \cdots, e_{N}^{T}\right]^{T}$. Then, $e$ satisfies

$$
\begin{aligned}
\dot{e}= & \left(I_{N-1} \otimes A\right) e+\mathcal{M} \sigma\left(\mathcal{S}\left(L_{1} \otimes I_{n}\right) e+c \Pi(e)\right) \\
& -\left(\mathbf{1}_{N-1} \otimes B\right) \sigma\left(u_{1}\right)
\end{aligned}
$$

where

$$
\begin{aligned}
& \mathcal{M}= \operatorname{diag}\left\{B\left(I-\rho_{2}\right), \ldots, B\left(I-\rho_{N}\right)\right\} \\
& \mathcal{S}= \operatorname{diag}\left\{K\left(\hat{\rho}_{2}\right), \ldots, K\left(\hat{\rho}_{N}\right)\right\} \\
& \Pi(e)=\left[\pi\left(-B^{T} P^{-1} \sum_{j=2}^{N} l_{2 j} e_{j}\right),\right. \\
&\left.\ldots, \pi\left(-B^{T} P^{-1} \sum_{j=2}^{N} l_{N j} e_{j}\right)^{T}\right]^{T} .
\end{aligned}
$$

Denote $\mathcal{H}(e)=\mathcal{S}\left(L_{1} \otimes I_{n}\right) e+c \Pi(e)$. Similarly, use $\mathcal{H}_{j}(e)$ to denote the $j$-th row of $\mathcal{H}(e)$ and define

$$
\wp^{*}(\mathcal{H}(e))=\left\{e \in \mathbb{R}^{n(N-1)}:\left|\mathcal{H}_{j}(e)\right| \leq 1\right\}
$$

Theorem 2: Under Assumptions 1 and 2, the leaderfollower consensus of agents described by (2) with $\rho_{1}=\mathbf{0}$ is reached by the controller (31) within the set $\varepsilon^{*}\left(L_{1} \otimes\right.$ $\left.P^{-1}, 1\right)$ if there exist a constant $k>0$ and matrices $P>$ $0, Q, E, F, G, Y_{0}, Y_{a h}, Y_{b h}$,

$$
\Theta^{i}=\left[\begin{array}{cc}
\Theta_{11}^{i} & \Theta_{12}^{i} \\
\left(\Theta_{12}^{i}\right)^{T} & \Theta_{22}^{i}
\end{array}\right]
$$

with $\Theta_{22}^{i}=\operatorname{diag}\left\{\Theta_{22}^{i 1}, \ldots, \Theta_{22}^{i m}\right\}, \Theta_{11}^{i} \in \mathbb{R}^{m n \times m n}, \Theta_{22}^{i p} \in$ $\mathbb{R}^{n \times n}, \quad \Theta_{22}^{i p} \leq 0(h, p \in \mathfrak{M}, i \in \mathcal{N})$ satisfying $\varepsilon^{*}\left(L_{1} \otimes\right.$ $\left.P^{-1}, 1\right) \subset \wp^{*}(\mathcal{H}(e))$ and the conditions given below:

$$
c\left(1-\max _{i, h, l}\left\{\bar{\rho}_{i h}^{l}\right\}\right) \geq \max \left\{\frac{1}{k \lambda_{\min }\left(L_{1}\right)}, \gamma_{1}\right\},
$$

$$
\begin{gathered}
A P+P A^{T}-\frac{2}{k} B B^{T}<0 \\
\Theta_{11}^{i}+\Theta_{12}^{i} \Delta\left(\hat{\rho}_{i}\right)+\left(\Theta_{12}^{i} \Delta\left(\hat{\rho}_{i}\right)\right)^{T}+\Delta\left(\hat{\rho}_{i}\right) \Theta_{22}^{i} \Delta\left(\hat{\rho}_{i}\right) \geq 0 \\
{\left[\begin{array}{cc}
Q & E \\
E^{T} & F
\end{array}\right]+G^{T} \Theta^{i} G<0} \\
\text { where } \hat{\rho}_{i} \in \Delta_{\hat{\rho}_{i}}, \rho_{i} \in\left\{\rho_{i}^{1}, \ldots, \rho_{i}^{l}\right\}, \rho_{i}^{q} \in N_{\rho_{i}^{q}}, \\
Q=\frac{1}{\lambda_{\max }\left(L_{1}\right)} A P+\left(\frac{1}{\lambda_{\max }\left(L_{1}\right)} A P\right)^{T}+B\left(I-\rho_{i}\right) Y_{0} \\
+\left(B\left(I-\rho_{i}\right) Y_{0}\right)^{T}+B \sum_{h=1}^{m} \rho_{i h} Y_{a h} \\
+\left(B \sum_{h=1}^{m} \rho_{i h} Y_{a h}\right)^{T}-\frac{2}{k \lambda_{\max }\left(L_{1}\right)} B B^{T}
\end{gathered}
$$

and $\Delta_{\hat{\rho}_{i}}, \Delta\left(\hat{\rho}_{i}\right), E, F, G, \hat{\rho}_{i h}$ are defined in accordance with Theorem 1. In addition, the feedback gains can be designed as

$$
K_{0}=Y_{0} P^{-1}, K_{a h}=Y_{a h} P^{-1}, K_{b h}=Y_{b h} P^{-1} .
$$

Proof: If $e \in \wp^{*}(H(e))$, then the system (32) becomes

$$
\dot{e}=\left(I_{N-1} \otimes A\right) e+\mathcal{M H}(e)-\left(\mathbf{1}_{N-1} \otimes B\right) u_{1} .
$$

According to [53] and the proof of Theorem 1, Theorem 2 can be readily proved.

Remark 3: In most results concerning the leader-follower consensus problem, the input of the leader has been assumed to be zero for simplicity. Such an assumption seems to be restrictive in many cases. For example, the nonzero input is often needed in order to avoid obstacles or threats. Also, the nonlinear part $\pi(\omega)$ in the controller (31) is often constructed for the follower to compensate for the effect on the consensus resulting from the nonzero input $u_{1}$ of the leader. The introduction of $\pi(\omega)$ adds more difficulties on the consensus analysis and DOA estimation problems. In the following part, the induced nonlinear constraint caused by $\pi(\omega)$ is first transformed into a linear constraint and then analyzed under the same structure as with the leaderless case.

\section{B. Domain of attraction}

Note from Theorem 2 that $\varepsilon^{*}\left(L_{1} \otimes P^{-1}, 1\right) \subset \wp^{*}(\mathcal{H}(e))$ holds if $\varepsilon\left(L_{1} \otimes P^{-1}, 1\right) \subset \wp^{*}(\mathcal{H}(e))$. Here, each row of $\mathcal{H}(e)$ can be calculated as

$$
\mathcal{H}_{(i-2) m+t}(e)= \begin{cases}\mathcal{H}_{(i-2) m+t}^{1}+\operatorname{sign}\left(\omega_{t}^{i}\right) c, & \omega_{t}^{i} \neq 0 \\ \mathcal{H}_{(i-2) m+t}^{2}, & \omega_{t}^{i}=0\end{cases}
$$

where $i \in \mathcal{N}, t \in \mathfrak{M}, \mathcal{H}^{1}=\left(\mathcal{S}-c I_{N-1} \otimes B^{T} P^{-1}\right)\left(L_{1} \otimes\right.$ $\left.I_{n}\right) e, \mathcal{H}^{2}=\mathcal{S}\left(L_{1} \otimes I_{n}\right) e$ and $\omega^{i}=-B^{T} P^{-1} \sum_{j=2}^{N} l_{i j} e_{j}$. The subscript denotes the corresponding row of the matrix or vector.

It follows from the inequality

$$
\left|\mathcal{H}_{(i-2) m+t}^{1}+\operatorname{sign}\left(\omega_{t}^{i}\right) c\right| \leq\left|\mathcal{H}_{(i-2) m+t}^{1}\right|+c
$$

that

$$
\left(\wp\left(\frac{1}{1-c} \mathcal{H}^{1}\right) \cap \wp\left(\mathcal{H}^{2}\right)\right) \subset \wp^{*}(\mathcal{H}(e)) .
$$


This article has been accepted for publication in a future issue of this journal, but has not been fully edited. Content may change prior to final publication. Citation information: DOI 10.1109/TSMC.2021.3050370, IEEE Transactions on Systems, Man, and Cybernetics: Systems

Finally, it can be inferred that $\varepsilon^{*}\left(L_{1} \otimes P^{-1}, 1\right) \subset \wp^{*}(\mathcal{H}(e))$ holds if

$$
\begin{aligned}
& \varepsilon\left(\frac{1}{\lambda_{\max }\left(L_{1}\right)} I_{N-1} \otimes P^{-1}, 1\right) \\
& \subset\left(\wp\left(\frac{1}{1-c}\left(\mathcal{S}-c I_{N-1} \otimes B^{T} P^{-1}\right)\right) \cap \wp(\mathcal{S})\right)
\end{aligned}
$$

holds. Furthermore, we know that (35) holds if and only if the following two inequalities are satisfied simultaneously for all $j=1,2, \ldots, m(N-1)$ :

$$
\begin{gathered}
{\left[\begin{array}{cc}
\frac{(1-c)^{2}}{2 \lambda_{\max }\left(L_{1}\right)} & \left(\mathcal{S}-c I_{N-1} \otimes B^{T} P^{-1}\right)_{j}\left(I_{N-1} \otimes P\right) \\
* & I_{N-1} \otimes P
\end{array}\right] \geq 0} \\
{\left[\begin{array}{cc}
\frac{1}{2 \lambda_{\max }\left(L_{1}\right)} & \mathcal{S}_{j}\left(I_{N-1} \otimes P\right) \\
* & I_{N-1} \otimes P
\end{array}\right] \geq 0 .}
\end{gathered}
$$

By means of the Schur Complement Lemma, (36) can be transformed into

$$
\begin{aligned}
& {\left[\begin{array}{cc}
\frac{-(1-c)^{2}}{2 \lambda_{\max }\left(L_{1}\right)} & -\left(Y_{0}-c B^{T}\right)_{j} \\
* & -P
\end{array}\right]} \\
& +\sum_{h=1}^{m} \hat{\rho}_{i h}\left[\begin{array}{cc}
0 & -\left(Y_{a h}+Y_{b h}\right)_{j} \\
* & \mathbf{0}
\end{array}\right] \leq 0
\end{aligned}
$$

and

$$
\begin{aligned}
& {\left[\begin{array}{cc}
\frac{-1}{2 \lambda_{\max }\left(L_{1}\right)} & -\left(Y_{0}\right)_{j} \\
* & -P
\end{array}\right]} \\
& +\sum_{h=1}^{m} \hat{\rho}_{i h}\left[\begin{array}{cc}
0 & -\left(Y_{a h}+Y_{b h}\right)_{j} \\
* & \mathbf{0}
\end{array}\right] \leq 0
\end{aligned}
$$

for all $\hat{\rho}_{i} \in \Delta_{\hat{\rho}_{i}}, i \in \mathcal{N}$ and $j \in \mathfrak{M}$.

In order to seek the largest DOA of the proposed controller, we introduce a prescribed ellipsoid $\epsilon\left(L_{1} \otimes R, 1\right)=\{z \in$ $\left.\mathbb{R}^{n(N-1)}: z^{T}\left(L_{1} \otimes R\right) z \leq 1\right\}$ and use $\max \alpha$ to estimate the DOA of the controller, where $\alpha$ satisfies

$$
\alpha \epsilon\left(L_{1} \otimes R, 1\right) \subset \varepsilon\left(L_{1} \otimes P^{-1}, 1\right) .
$$

Finally, we arrive at the following optimization problem:

$$
\max _{\hat{\rho}_{i}, \rho_{i}, k, P, Y_{0}, Y_{a}, Y_{b}} \alpha \text {, s.t. (34), (37), (38), }
$$

which can be rewritten as

$$
\min _{\hat{\rho}_{i}, \rho_{i}, k, P, Y_{0}, Y_{a}, Y_{b}} \gamma, \quad \text { s.t. (34), (37), } R^{-1} \leq 2 \gamma P .
$$

where $\gamma=\alpha^{-2}$.

Remark 4: From the above analysis of the DOA, we use the inequality

$$
\left|\mathcal{H}_{(i-2) m+t}^{1}+\operatorname{sign}\left(\omega_{t}^{i}\right) c\right| \leq\left|\mathcal{H}_{(i-2) m+t}^{1}\right|+c \leq 1
$$

to relax the restriction so that $\left(\wp\left(\frac{1}{1-c} \mathcal{H}^{1}\right) \cap \wp\left(\mathcal{H}^{2}\right)\right) \subset$ $\wp^{*}(\mathcal{H}(e))$ can be established. If $c \geq 1$, then $\wp\left(\frac{1}{1-c} \mathcal{H}^{1}\right)=$ $\varnothing$ or 0 . As a result, we cannot find a suitable $P>0$ to ensure $\varepsilon^{*}\left(L_{1} \otimes P^{-1}, 1\right) \subset\left(\wp\left(\frac{1}{1-c} \mathcal{H}^{1}\right) \cap \wp\left(\mathcal{H}^{2}\right)\right)$. Therefore, $c<1$ is a necessary condition in our proposed method.

Remark 5: For the DOA, we would like to clarify that: 1) the DOA is introduced to optimize the controller, that is, the DOA works as an index in the optimization of the controller gains, and typically, we hope to find the largest DOA; 2) according to Definition 1, if and only if the initial states of each agent are contained in the DOA, the consensus of agents can be achieved by the designed controller; 3$)$ in this paper, $\varepsilon\left(\Phi \otimes P^{-1}, 1\right)$ for the leaderless case or $\varepsilon\left(L_{1} \otimes P^{-1}, 1\right)$ for the leader-follower case has proven to be a subset of the DOA; and 4) even if the initial states lie outside $\varepsilon\left(\Phi \otimes P^{-1}, 1\right)$ or $\varepsilon\left(L_{1} \otimes P^{-1}, 1\right)$, the leaderless or leader-follower consensus may still be reached.

\section{Simulation}

Example 1: (Leaderless consensus) Consider an MAS consisting of five agents described by (1) with

$$
A=\left[\begin{array}{cc}
0.6 & -0.8 \\
0.8 & 0.6
\end{array}\right], \quad B=\left[\begin{array}{l}
2 \\
4
\end{array}\right],
$$

where $A$ is unstable but $(A, B)$ is stabilizable. Fig. 1(a) depicts the undirected topology. Consider the following two modes $(i=1, \ldots, 5)$ :

(1) All agents are normally operational with $\rho_{i}^{1}=0$.

(2) The fault of loss-of-actuation-effectiveness takes place with $0<\rho_{i}^{2} \leq 0.6$.

In order to make comparisons, let us design the following three kinds of controllers:

1) proposed controller in this paper: the proposed controller in this paper with consideration of actuator saturations;

2) fixed-gain controller 1: the fixed-gain controller proposed in [11] with consideration of actuator saturations;

3) fixed-gain controller 2: the fixed-gain controller designed satisfying (9a), (9b), (29) and (30) without consideration of actuator saturations.

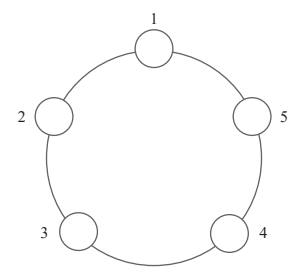

(a) Leaderless

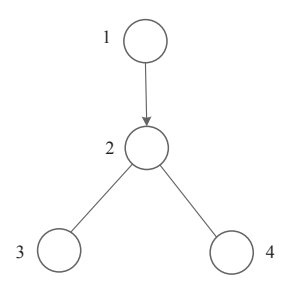

(b) Leader-follower
Fig. 1. Topology graphs.

Case (1): Proposed controller in this paper Let $R=\left[\begin{array}{ll}1 & 0 \\ 0 & 1\end{array}\right]$. By Theorem 1, we have

$$
\begin{aligned}
P & =\left[\begin{array}{ll}
0.1946 & 0.0960 \\
0.0960 & 0.1386
\end{array}\right], \\
K_{0} & =\left[\begin{array}{ll}
0.0748 & -0.4112
\end{array}\right], \\
K_{a} & =\left[\begin{array}{ll}
0.1092 & -0.5996
\end{array}\right], \\
K_{b} & =\left[\begin{array}{ll}
-0.1219 & 0.6690
\end{array}\right], \\
k=100, c & =0.0191, t_{0}=0.1, \alpha=0.365 .
\end{aligned}
$$

Case (2): Fixed-gain controller 1

By Theorem 1 in [11], we have 
This article has been accepted for publication in a future issue of this journal, but has not been fully edited. Content may change prior to final publication. Citation information: DOI 10.1109/TSMC.2021.3050370, IEEE Transactions on Systems, Man, and Cybernetics: Systems

$$
\begin{aligned}
P_{f_{1}} & =\left[\begin{array}{ll}
0.1946 & 0.0960 \\
0.0960 & 0.1386
\end{array}\right], \\
K_{0 f_{1}} & =\left[\begin{array}{ll}
0.0585 & -0.3225
\end{array}\right], \\
k_{f_{1}}=100, & c_{f_{1}}=0.0191, \alpha_{f_{1}}=0.365 .
\end{aligned}
$$

Case (3): Fixed-gain controller 2

From (9a), (9b), (29) and (30), we have

$$
\begin{aligned}
P_{f_{2}} & =\left[\begin{array}{ll}
0.2230 & 0.1584 \\
0.1584 & 0.1306
\end{array}\right], \\
K_{0 f_{2}} & =\left[\begin{array}{ll}
5.2916 & -8.1700
\end{array}\right], \\
k_{f_{2}} & =50, c_{f_{2}}=0.1362 .
\end{aligned}
$$

Let $\rho_{1}=0.4, \quad \rho_{2}=0.2, \quad \rho_{3}=0.08, \quad \rho_{4}=0.32$ and $\rho_{5}=0.36$. Suppose that the initial state is $x(0)=$ $\left[\begin{array}{llllllllll}-0.5 & 0 & 0 & 0.3 & 0 & 0 & 0.6 & 0 & 0 & -0.4\end{array}\right]^{T}$. Simulation results with the above three kinds of controllers are shown in Figs. 2-5. Figs. 2-3 correspond to the proposed controller in this paper, Fig. 4 corresponds to the fixed-gain controller 1 and Fig. 5 corresponds to the fixed-gain controller 2. We would like to mention that as shown in Fig. 3(b), the adaptive parameter $\hat{\rho}_{i}$ has a deviation from the actual $\rho_{i}$. This is reasonable because Theorem 1 cannot guarantee that the estimated error of $\rho_{i}$ converges to zero. Compared with the fixed-gain controller 1 , the proposed controller indeed exhibits better dynamic performance, which exactly benefits from the adaptive structure. Besides, the serious chattering phenomenon in Fig. 5(b) indicates that actuator saturations, if not appropriately handled, could have a dramatic effect on the system.
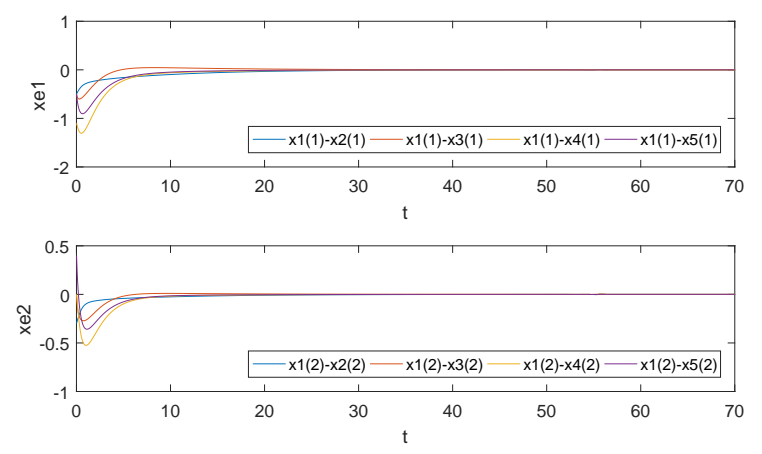

Fig. 2. Consensus errors of the state variables with the proposed controller.

Example 2: (Leader-follower consensus) Consider an MAS consisting of four UAVs, Silver Fox, whose linear lateral dynamics can be described by (1) with [49]

$$
\begin{gathered}
A=\left[\begin{array}{ccc}
-0.1798 & 0.069 & -0.9976 \\
-22.4565 & -8.213 & 2.0046 \\
15.0747 & -0.6578 & -0.7095
\end{array}\right], \\
B=\left[\begin{array}{cc}
0 & 0.0873 \\
99.5144 & 2.4034 \\
-7.9397 & -10.1124
\end{array}\right] .
\end{gathered}
$$

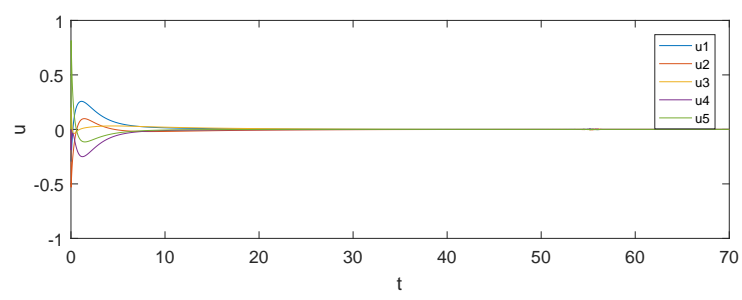

(a) Trajectories of the input variables

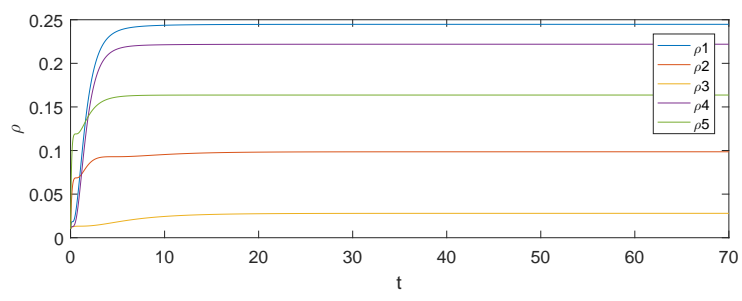

(b) Trajectories of the adaptive parameters

Fig. 3. Input and adaptive parameter trajectories with the proposed controller.
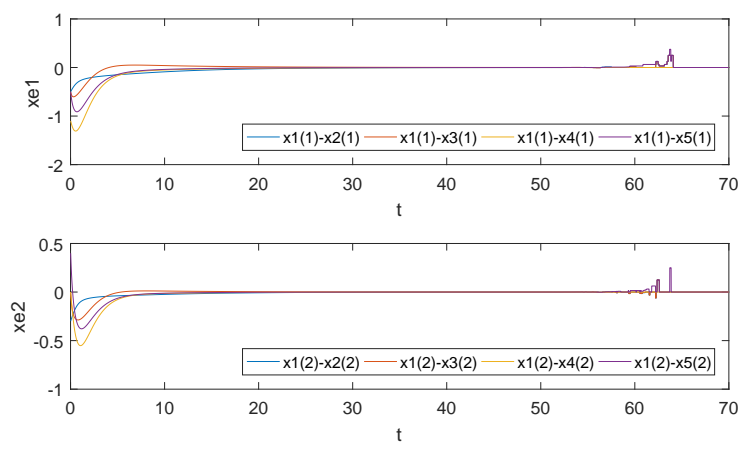

(a) Consensus errors of the state variables

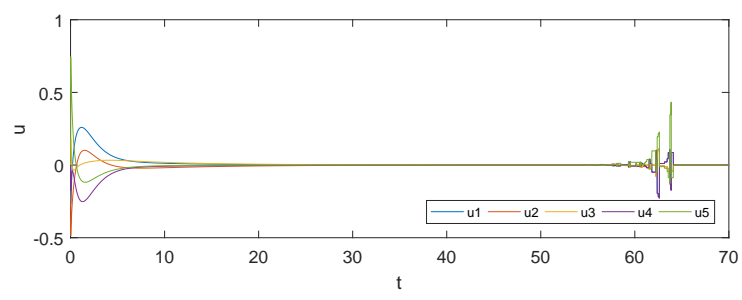

(b) Trajectories of the input variables

Fig. 4. Consensus errors and input trajectories with the fixed-gain controller 1 .

The topology of the underlying MAS is shown in Fig. 1(b). Agent 1 is the leader with dynamics

$$
\left\{\begin{array}{l}
\dot{x}_{1}=A x_{1}+B \sigma\left(u_{1}\right) \\
u_{1}=K_{l} x \\
x_{1}(0)=\left[\begin{array}{lll}
1 & 0 & 0
\end{array}\right]^{T} .
\end{array}\right.
$$

where

$$
K_{l}=\left[\begin{array}{ccc}
-0.0494 & 0.2398 & -0.0894 \\
0.0269 & -0.0048 & -0.2466
\end{array}\right]
$$


This article has been accepted for publication in a future issue of this journal, but has not been fully edited. Content may change prior to final publication. Citation information: DOI 10.1109/TSMC.2021.3050370, IEEE Transactions on Systems, Man, and Cybernetics: Systems
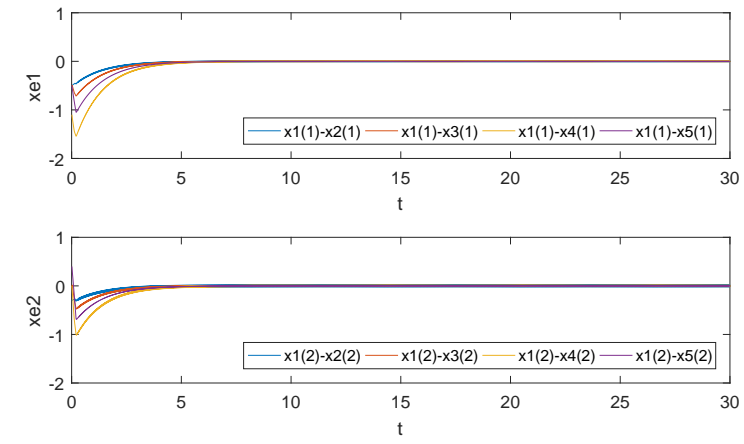

(a) Consensus errors of the state variables

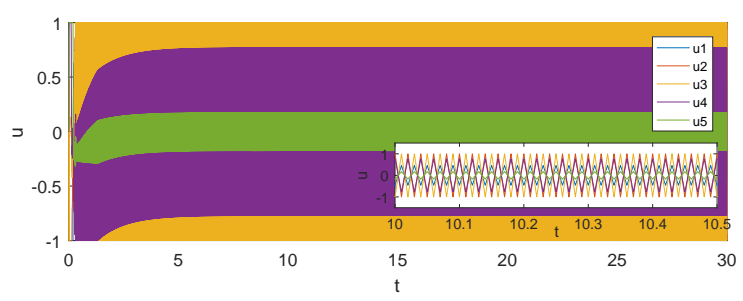

(b) Trajectories of the input variables

Fig. 5. Consensus errors and input trajectories with the fixed-gain controller 2.

For all the followers, we consider the same modes shown in Example 1. By Theorem 2, we have

$$
\begin{aligned}
& K_{0}=\left[\begin{array}{lll}
-0.00050 & -0.00003 & 0.00017 \\
-0.00008 & -0.00001 & 0.00006
\end{array}\right], \\
& K_{a}=\left[\begin{array}{cccc}
-0.0698 & -0.0127 & 0.0250 & -0.0272 \\
0.4474 & 0.0872 & -0.0263 & -9.7618
\end{array}\right. \\
& \left.\begin{array}{cc}
-0.0064 & -0.0189 \\
-1.5242 & 7.8564
\end{array}\right] \\
& K_{b}=\left[\begin{array}{cccc}
0.0703 & 0.0127 & -0.0252 & -0.3702 \\
-0.4474 & -0.0872 & 0.0263 & -5.5999
\end{array}\right. \\
& \left.\begin{array}{ll}
-0.0710 & 0.0047 \\
-0.8069 & 5.4646
\end{array}\right] \text {, } \\
& k=100, c=0.1250, t_{0}=10, \alpha=163.737 .
\end{aligned}
$$

Let $\rho_{2}=\operatorname{diag}\{0.4,0.32\}, \quad \rho_{3}=\operatorname{diag}\{0.2,0.12\}$ and $\rho_{4}=\operatorname{diag}\{0.08,0.16\}$. The initial states of followers are $x(0)=\left[\begin{array}{lllllllll}0 & -1 & 0 & 1 & 0 & -1 & 0 & -2 & 0\end{array}\right]^{T}$. The simulation result is shown in Figs. 6-7. The leader-follower consensus with actuator faults is achieved, see Fig. 6. However, the undesirable chattering phenomenon (see Fig. 7) caused by the discontinuous controller is harmful to the system. Inspired by [23], we design a continuous controller to avoid the chattering phenomenon with

$$
\pi\left(\omega_{t}\right)=\left\{\begin{array}{ll}
\omega_{t}+\operatorname{sign}\left(\omega_{t}\right) c, & \left|c \omega_{t}\right|>\kappa \\
\omega_{t}+\frac{c \omega_{t}}{\kappa}, & \left|c \omega_{t}\right| \leq \kappa
\end{array} .\right.
$$

The simulation result with $\kappa=0.1$ is shown in Figs. 8-9, which implies that the chattering phenomenon is avoided.
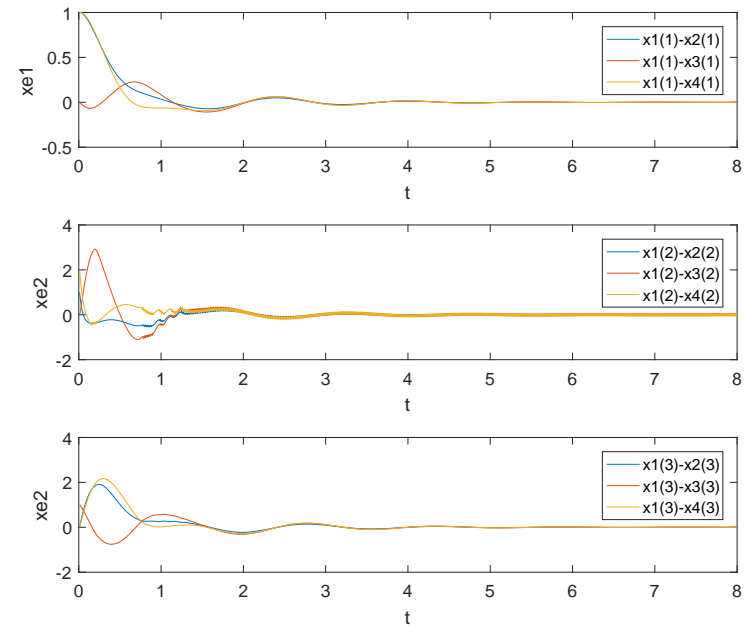

Fig. 6. Leader-follower consensus errors with the discontinuous controller.
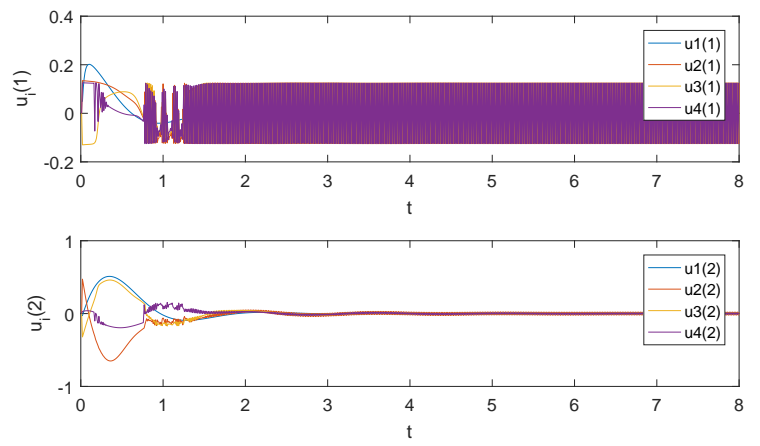

Fig. 7. Input trajectories of the leader-follower MAS with the discontinuous controller.
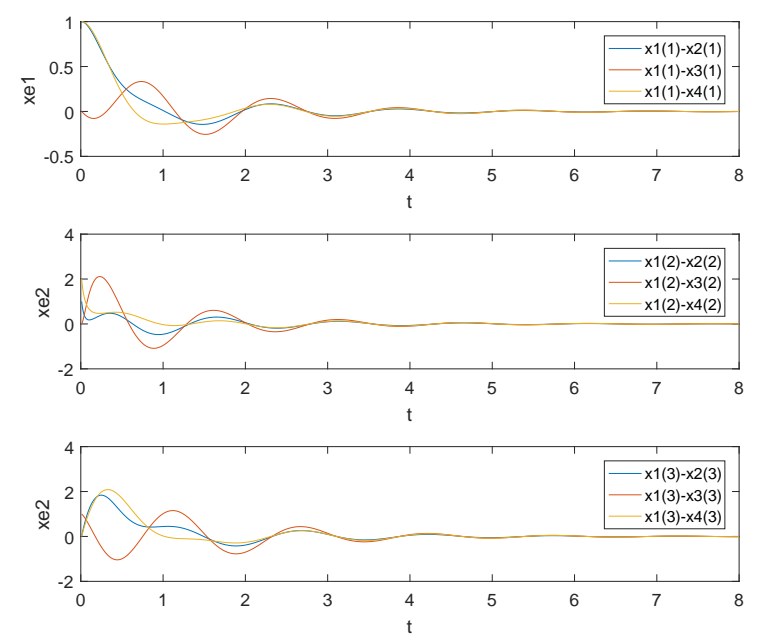

Fig. 8. Leader-follower consensus errors with the continuous controller.

\section{CONCLUSION}

In this paper, we have proposed a kind of saturationresistant fault-tolerant consensus method for a group of gener- 
This article has been accepted for publication in a future issue of this journal, but has not been fully edited. Content may change prior to final publication. Citation information: DOI 10.1109/TSMC.2021.3050370, IEEE Transactions on Systems, Man, and Cybernetics: Systems
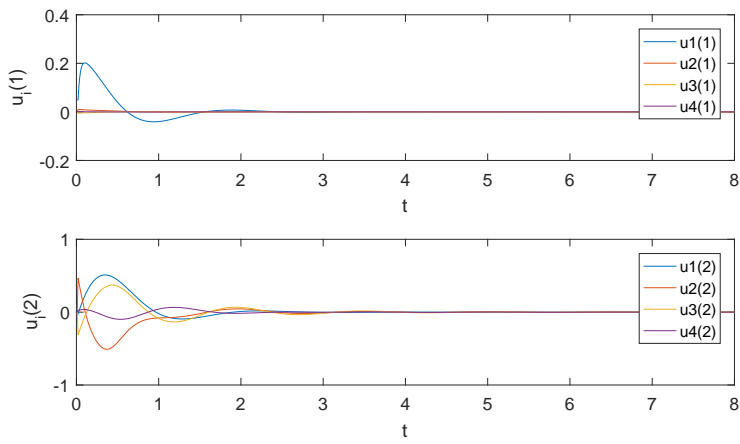

Fig. 9. Input trajectories of the leader-follower MAS with the continuous controller.

al linear MASs. For the leaderless and leader-follower MASs, a consensus controller has been designed to guarantee the consensus in the presence of actuator faults, and such a controller has then been optimized by solving an established optimization problem composed of several LMIs. Finally, simulation results have been presented to illustrate the effectiveness of the proposed controller. The method of dealing with actuator saturations in this paper, i.e. the saturation-resistant method, can be used in many control problems such as flocking control, formation control and pinning control for complex systems [2], [3], [27], [35], [48], [51]. In the future research, the outage fault together with the control allocation issue is one of our focuses. Besides, we are interested in the switching topologies [14], [22], which also deserves further investigation especially when confronted with actuator saturations.

\section{REFERENCES}

[1] H. Bang, M.-J. Tahk and H.-D. Choi, Large angle attitude control of spacecraft with actuator saturation, Control Engineering Practice, vol. 11, no. 9, pp. 989-997, 2003.

[2] M. Basin and F. G. Avellaneda, Continuous fixed-time controller design for dynamic systems with unmeasurable states subject to unbounded disturbances, Asian Journal of Control, vol. 21, no. 1, pp. 194-207, 2019.

[3] R. Caballero-Aguila, A. Hermoso-Carazo and J. Linares-Perez, Distributed fusion filters from uncertain measured outputs in sensor networks with random packet losses, Information Fusion, vol. 34: 70-79, 2017.

[4] L. Cheng, Y. Wang, W. Ren, Z. G. Hou and M. Tan, On convergence rate of leader-following consensus of linear multi-agent systems with communication noises, IEEE Transactions on Automatic Control, vol. 61, no. 11, pp. 3586-3592, 2016.

[5] Y. Cui, Y. Liu, W. Zhang and F. E. Alsaadi, Sampled-based consensus for nonlinear multiagent systems with deception attacks: The decoupled method, IEEE Transactions on Systems, Man, and Cybernetics: Systems, in press, DOI: 10.1109/TSMC.2018.2876497.

[6] M. Davoodi, N. Meskin and K. Khorasani, Simultaneous fault detection and consensus control design for a network of multi-agent systems, Automatica, vol. 66, pp. 185-194, 2016.

[7] S. M. Dibaji and H. Ishii, Resilient consensus of second-order agent networks: Asynchronous update rules with delays, Automatica, vol. 81, pp. 123-132, 2017.

[8] D. Ding, Z. Wang, B. Shen and G. Wei, Event-triggered consensus control for discrete-time stochastic multi-agent systems: The input-tostate stability in probability, Automatica, vol. 62, pp. 284-291, 2015.

[9] D. Ding, Z. Wang and Q.-L. Han, Neural-network-based consensus control for multiagent systems with input constraints: The event-triggered case, IEEE Transactions on Cybernetics, vol. 50, no. 8, pp. 3719-3730, 2020.
[10] J. A. Fax and R. M. Murray, Graph laplacians and stabilization of vehicle formations, In: Proc. 15th IFAC World Congress, vol. 35, no. 1, pp. 5560, 2002.

[11] C. Gao and X. He, Fault-tolerant consensus control for multi-agent systems with actuator saturation, In: Proc. 33rd Youth Academic Annual Conference of Chinese Association of Automation (YAC), pp. 484-488, 2018.

[12] C. Gao, Z. Wang, X. He and Q.-L. Han, On consensus of second-order multiagent systems with actuator saturations: A generalized-Nyquistcriterion-based approach, IEEE Transactions on Cybernetics, in press, DOI: $10.1109 /$ TCYB.2020.3025824.

[13] R. A. Horn and C. R. Johnson, Matrix analysis, United Kingdom: Cambridge University Press, 1985.

[14] J. Hu, Z. Wang, G.-P. Liu, C. Jia and J. Williams, Event-triggered recursive state estimation for dynamical networks under randomly switching topologies and multiple missing measurements, Automatica, vol. 115, art. no. $108908,2020$.

[15] T. Hu and Z. Lin, Control systems with actuator saturation: Analysis and design, Boston: Birkhäuser, 2001.

[16] T. Hu, Z. Lin and B. M. Chen, An analysis and design method for linear systems subject to actuator saturation and disturbance, Automatica, vol. 38, no. 2, pp. 351-359, 2002.

[17] Y. Jia, J. Du, W. Zhang and L. Wang, Three-dimensional leaderless flocking control of large-scale small unmanned aerial vehicles, In: Proc. 20th IFAC World Congress, vol. 50, no. 1, pp. 6208-6213, 2017.

[18] H. K. Khalil, Nonlinear Systems, Englewood Cliffs, New Jersey: Prentice Hall, 2002.

[19] M. Khalili, X. Zhang, M. M. Polycarpou, T. Parisini and Y. Cao, Distributed adaptive fault-tolerant control of uncertain multi-agent systems, Automatica, vol. 87, pp. 142-151, 2018.

[20] H. J. LeBlanc, H. Zhang, X. Koutsoukos and S. Sundaram, Resilient asymptotic consensus in robust networks, IEEE Journal on Selected Areas in Communications, vol. 31, no. 4, pp. 766-781, 2013.

[21] B. Li, Z. Wang, Q.-L. Han and H. Liu, Distributed quasiconsensus control for stochastic multiagent systems under Round-Robin protocol and uniform quantization, IEEE Transactions on Cybernetics, in press, DOI: $10.1109 /$ TCYB.2020.3026001.

[22] Q. Li, Z. Wang, N. Li and W. Sheng, A dynamic event-triggered approach to recursive filtering for complex networks with switching topologies subject to random sensor failures, IEEE Transactions on Neural Networks and Learning Systems, vol. 31. no. 10, pp. 4381-4388, 2020.

[23] Z. Li, W. Ren, X. Liu and L. Xie, Distributed consensus of linear multiagent systems with adaptive dynamic protocols, Automatica, vol. 49, no. 7, pp. 1986-1995, 2013.

[24] Z. Lin and A. Saberi, Semi-global exponential stabilization of linear systems subject to "input saturation" via linear feedbacks, Systems \& Control Letters, vol. 21, no. 3, pp. 225-239, 1993.

[25] Z. Lin and A. Saberi, A semi-global low-and-high gain design technique for linear systems with input saturation-stabilization and disturbance rejection, International Journal of Robust and Nonlinear Control, vol. 5, no. 5, pp. 381-398, 1995

[26] Z. Lin, Low gain feedback, London: Springer, 1998

[27] Y. Liu, Z. Wang, L. Ma and F. E. Alsaadi, A partial-nodes-based information fusion approach to state estimation for discrete-time delayed stochastic complex networks, Information Fusion, vol. 49, pp. 240-248, Sept. 2019.

[28] Q. Liu, Z. Wang, X. He and D. H. Zhou, On Kalman-consensus filtering with random link failures over sensor networks, IEEE Transactions on Automatic Control, vol. 63, no. 8, pp. 2701-2708, 2018.

[29] Z. Meng, Z. Zhao and Z. Lin, On global leader-following consensus of identical linear dynamic systems subject to actuator saturation, Systems \& Control Letters, vol. 62, no. 2, pp. 132-142, 2013

[30] R. Olfati-Saber and R. M. Murray, Consensus protocols for networks of dynamic agents, In: Proc. 2003 American Control Conference, pp. 951956, 2003.

[31] R. Olfati-Saber and R. M. Murray, Consensus problems in networks of agents with switching topology and time-delays, IEEE Transactions on Automatic Control, vol. 49, no. 9, pp. 1520-1533, 2004.

[32] C. Peng, J. Zhang and Q.-L. Han, Consensus of multiagent systems with nonlinear dynamics using an integrated sampled-data-based eventtriggered communication scheme, IEEE Transactions on Systems, Man, and Cybernetics-Systems, vol. 49, no. 3, pp. 589-599, 2019.

[33] J. Qi, S. Wang, J.-A. Fang and M. Diagne, Control of multi-agent systems with input delay via PDE-based method, Automatica, vol. 106, pp. 91-100, 2019. 
This article has been accepted for publication in a future issue of this journal, but has not been fully edited. Content may change prior to final publication. Citation information: DOI 10.1109/TSMC.2021.3050370, IEEE Transactions on Systems, Man, and Cybernetics: Systems FINAL VERSION

[34] R. Sakthivel, A. Parivallal, B. Kaviarasan, H. Lee and Y. Lim, Finite-time consensus of Markov jumping multi-agent systems with time-varying actuator faults and input saturation, ISA Transactions, vol. 83, pp. 8999, 2018.

[35] B. Shen, Z. Wang, D. Wang and Q. Li, State-saturated recursive filter design for stochastic time-varying nonlinear complex networks under deception attacks, IEEE Transactions on Neural Networks and Learning Systems, vol. 31, no. 10, pp. 3788-3800, 2020.

[36] H. Su, M. Z. Chen, X. Wang and J. Lam, Semiglobal observer-based leader-following consensus with input saturation, IEEE Transactions on Industrial Electronics, vol. 61, no. 6, pp. 2842-2850, 2014.

[37] H. Su, Y. Ye, Y. Qin, Y. Cao and M. Z. Chen, Semi-global output consensus for discrete-time switching networked systems subject to input saturation and external disturbances, IEEE transactions on cybernetics, vol. 49, no. 11 , pp. $3934-3945,2019$.

[38] J. Thunberg, J. Goncalves and X. Hu, Consensus and formation control on $S E(3)$ for switching topologies, Automatica, vol. 66, pp. 109-121, 2016.

[39] N. Wan, D. S. Naidu, M. Liu, L. Wu and W. Yao, Adaptive sliding mode control for spacecraft rendezvous in near-circular orbits with time-varying saturation constraint, In: Proc. 2017 American Control Conference (ACC), pp. 5812-5817, 2017.

[40] L. Wang, Z. Wang, Q.-L. Han and G. Wei, Synchronization control for a class of discrete-time dynamical networks with packet dropouts: A coding-decoding-based approach, IEEE Transactions on Cybernetics, vol. 48, no. 8, pp. 2437-2448, 2018.

[41] X. Wang, S. Li and J. Lam, Distributed active anti-disturbance output consensus algorithms for higher-order multi-agent systems with mismatched disturbances, Automatica, vol. 74, pp. 30-37, 2016.

[42] B. Xiao, Q. Hu and Y. Zhang, Adaptive sliding mode fault tolerant attitude tracking control for flexible spacecraft under actuator saturation, IEEE Transactions on Control Systems Technology, vol. 20, no. 6 , pp. 1605-1612, 2012.

[43] W. Xu, Z. Wang and D. W. C. Ho, Finite-horizon $H_{\infty}$ consensus for multiagent systems with redundant channels via an observer-type eventtriggered scheme, IEEE Transactions on Cybernetics, vol. 48, no. 5, pp. 1567-1576, 2018

[44] G.-H. Yang and D. Ye, Reliable $H_{\infty}$ control of linear systems with adaptive mechanism, IEEE Transactions on Automatic Control, vol. 55, no. 1, pp. 242-247, 2010.

[45] T. Yang, Z. Meng, D. V. Dimarogonas and K. H. Johansson, Global consensus for discrete-time multi-agent systems with input saturation constraints, Automatica, vol. 50, no. 2, pp. 499-506, 2014.

[46] X. You, C. Hua and X. Guan, Event-triggered leader-following consensus for nonlinear multiagent systems subject to actuator saturation using dynamic output feedback method. IEEE Transactions on Automatic Control, vol. 63, no. 12, pp. 4391-4396, 2018

[47] Y. Yuan, Z. Wang, P. Zhang and H. Dong, Nonfragile near-optimal control of stochastic time-varying multi-agent systems with control- and state-dependent noises, IEEE Transactions on Cybernetics, vol. 49, no. 7 , pp. 2605-2617, 2019.

[48] Z. Zhao, Z. Wang, L. Zou and J. Guo, Set-Membership filtering for timevarying complex networks with uniform quantisations over randomly delayed redundant channels, International Journal of Systems Science, in press, DOI: $10.1080 / 00207721.2020 .1814898$.

[49] Z. Zhen, G. Tao, Y Xu and G. Song, Multivariable adaptive control based consensus flight control system for UAVs formation, Aerospace Science and Technology, vol. 93, pp. 105336, 2019.

[50] B. Zhou, W. Wang and H. Ye, Cooperative control for consensus of multi-agent systems with actuator faults, Computers \& Electrical Engineering, vol. 40, no. 7, pp. 2154-2166, 2014.

[51] L. Zou, Z. Wang, H. Dong and Q.-L. Han, Moving horizon estimation with multirate measurements and correlated noises, International Journal of Robust and Nonlinear Control, vol. 30, no. 17, pp. 7429-7445, 2020.

[52] Z. Zuo and Y. Wang, On enlarging the domain of attraction for linear systems subject to actuator saturation, International Journal of General Systems, vol. 37, no. 2, pp. 239-248, 2008.

[53] Z. Zuo, J. Zhang and Y. Wang, Adaptive fault-tolerant tracking control for linear and lipschitz nonlinear multi-agent systems, IEEE Transactions on Industrial Electronics, vol. 62, no. 6, pp. 3923-3931, 2015.

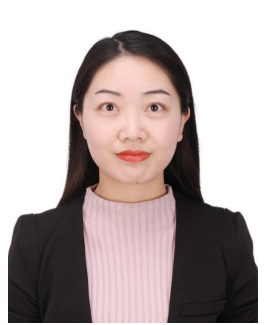

Chen Gao received the M.Eng. degree in navigation, guidance and control from the Nanjing University of Aeronautics and Astronautics, Nanjing, China, in 2016.

In 2016, she joined the Tsinghua University, where she is currently working towards a Ph.D. degree in control science and engineering. From March 2019 to August 2019, she was a visiting scholar with the Department of Computer Science, Brunel University London, London, U.K. Her research interests include fault diagnosis, fault-tolerant control

and multi-agent systems

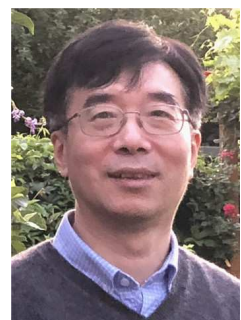

Zidong Wang (SM'03-F'14) was born in Jiangsu, China, in 1966. He received the B.Sc. degree in mathematics in 1986 from Suzhou University, Suzhou, China, and the M.Sc. degree in applied mathematics in 1990 and the Ph.D. degree in electrical engineering in 1994, both from Nanjing University of Science and Technology, Nanjing, China.

$\mathrm{He}$ is currently Professor of Dynamical Systems and Computing in the Department of Computer Science, Brunel University London, U.K. From 1990 to 2002, he held teaching and research appointments in universities in China, Germany and the UK. Prof. Wang's research interests include dynamical systems, signal processing, bioinformatics, control theory and applications. He has published more than 600 papers in international journals. He is a holder of the Alexander von Humboldt Research Fellowship of Germany, the JSPS Research Fellowship of Japan, William Mong Visiting Research Fellowship of Hong Kong.

Prof. Wang serves (or has served) as the Editor-in-Chief for International Journal of Systems Science, the Editor-in-Chief for Neurocomputing, and an Associate Editor for 12 international journals including IEEE Transactions on Automatic Control, IEEE Transactions on Control Systems Technology, IEEE Transactions on Neural Networks, IEEE Transactions on Signal Processing, and IEEE Transactions on Systems, Man, and Cybernetics-Part C. He is a Member of the Academia Europaea, a Fellow of the IEEE, a Fellow of the Royal Statistical Society and a member of program committee for many international conferences.

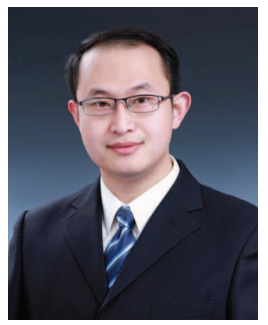

Xiao He (M'12-SM'20) received the B.Eng. degree in information technology from the Beijing Institute of Technology, Beijing, China, in 2004, and the $\mathrm{Ph} . \mathrm{D}$. degree in control science and engineering from the Tsinghua University, Beijing, China, in 2010.

$\mathrm{He}$ is currently a tenured Associate Professor with the Department of Automation, Tsinghua University. He has authored more than 60 papers in refereed international journals. His research interests include fault diagnosis and fault-tolerant control, networked systems, cyber-physical systems, as well as their

applications

Dr. He is now a Full Member of Sigma Xi Scientific Research Society, a Senior Member of the Chinese Association of Automation, and a Senior Member of the IEEE. He is an Associate Editor of the Control Engineering Practice. 
This article has been accepted for publication in a future issue of this journal, but has not been fully edited. Content may change prior to final publication Citation information: DOI 10.1109/TSMC.2021.3050370, IEEE Transactions on Systems, Man, and Cybernetics: Systems

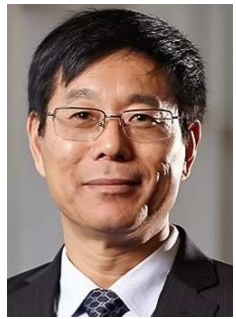

Qing-Long Han received the B.Sc. degree in Mathematics from Shandong Normal University, Jinan, China, in 1983, and the M.Sc. and Ph.D. degrees in Control Engineering from East China University of Science and Technology, Shanghai, China, in 1992 and 1997, respectively.

Professor Han is Pro Vice-Chancellor (Research Quality) and a Distinguished Professor at Swinburne University of Technology, Melbourne, Australia. He held various academic and management positions at Griffith University and Central Queensland University, Australia. His research interests include networked control systems, multiagent systems, time-delay systems, smart grids, unmanned surface vehicles, and neural networks. Professor Han is a Highly Cited Researcher according to Clarivate Analytics. He is a Fellow of The Institution of Engineers Australia. $\mathrm{He}$ is one of Australia's Top 5 Lifetime Achievers (Research Superstars) in the discipline area of Engineering and Computer Science by The Australians 2020 Research Magazine. He received the 2020 IEEE Systems, Man, and Cybernetics Society Andrew P. Sage Best Transactions Paper Award, the 2020 IEEE Industrial Electronics Society IEEE Transactions on Industrial Informatics Outstanding Paper Award, and the 2019 IEEE Systems, Man, and Cybernetics Society Andrew P. Sage Best Transactions Paper Award.

Professor Han is Co-Editor of Australian Journal of Electrical and Electronic Engineering, an Associate Editor for 12 international journals, including the IEEE TRANSACTIONS ON CYBERNETICS, the IEEE TRANSACTIONS ON INDUSTRIAL INFORMATICS, IEEE INDUSTRIAL ELECTRONICS MAGAZINE, the IEEE/CAA JOURNAL OF AUTOMATICA SINICA, Control Engineering Practice, and Information Sciences, and a Guest Editor for 13 Special Issues. 